\title{
Computational drug repurposing against SARS-CoV-2 reveals plasma membrane cholesterol depletion as key factor of antiviral drug activity
}

\author{
Szilvia Barsi ${ }^{1}$, Henrietta Papp ${ }^{2,3}$, Alberto Valdeolivas Urbelz ${ }^{4}$, Dániel J. Tóth ${ }^{1}$, Anett Kuczmog ${ }^{2,3}$, \\ Mónika Madai ${ }^{2,3}$, László Hunyady ${ }^{1,5}$, Péter Várnai ${ }^{1,5}$, Julio Saez-Rodriguez ${ }^{4,6}$, Ferenc Jakab ${ }^{2,3}$, \\ Bence Szalai ${ }^{1, \$, *}$
}

${ }^{1}$ Semmelweis University, Faculty of Medicine, Department of Physiology, Budapest, Hungary

${ }^{2}$ National Laboratory of Virology, Szentágothai Research Centre, University of Pécs, Pécs, Hungary

${ }^{3}$ Institute of Biology, Faculty of Sciences, University of Pécs, 7624 Pécs, Hungary

${ }^{4}$ Heidelberg University, Faculty of Medicine, and Heidelberg University Hospital, Institute for Computational Biomedicine, Bioquant, Heidelberg, Germany

${ }^{5}$ MTA-SE Laboratory of Molecular Physiology, Budapest, Hungary

$\$$ Current address: Turbine Ltd., Budapest, Hungary

*Corresponding author: ben.szalai.cb@gmail.com 


\begin{abstract}
Comparing SARS-CoV-2 infection-induced gene expression signatures to drug treatment-induced gene expression signatures is a promising bioinformatic tool to repurpose existing drugs against SARS-CoV-2. The general hypothesis of signature based drug repurposing is that drugs with inverse similarity to a disease signature can reverse disease phenotype and thus be effective against it. However, in the case of viral infection diseases, like SARS-CoV-2, infected cells also activate adaptive, antiviral pathways, so that the relationship between effective drug and disease signature can be more ambiguous.

To address this question, we analysed gene expression data from in vitro SARS-CoV-2 infected cell lines, and gene expression signatures of drugs showing anti-SARS-CoV-2 activity. Our extensive functional genomic analysis showed that both infection and treatment with in vitro effective drugs leads to activation of antiviral pathways like NFkB and JAK-STAT. Based on the similarity - and not inverse similarity - between drug and infection-induced gene expression signatures, we were able to predict the in vitro antiviral activity of drugs. We also identified SREBF1/2, key regulators of lipid metabolising enzymes, as the most activated transcription factors by several in vitro effective antiviral drugs. Using a fluorescently labeled cholesterol sensor, we showed that these drugs decrease the cholesterol levels of plasma-membrane. Supplementing drug-treated cells with cholesterol reversed the in vitro antiviral effect, suggesting the depleting plasma-membrane cholesterol plays a key role in virus inhibitory mechanism.
\end{abstract}

Our results can help to more effectively repurpose approved drugs against SARS-CoV-2, and also highlights key mechanisms behind their antiviral effect. 


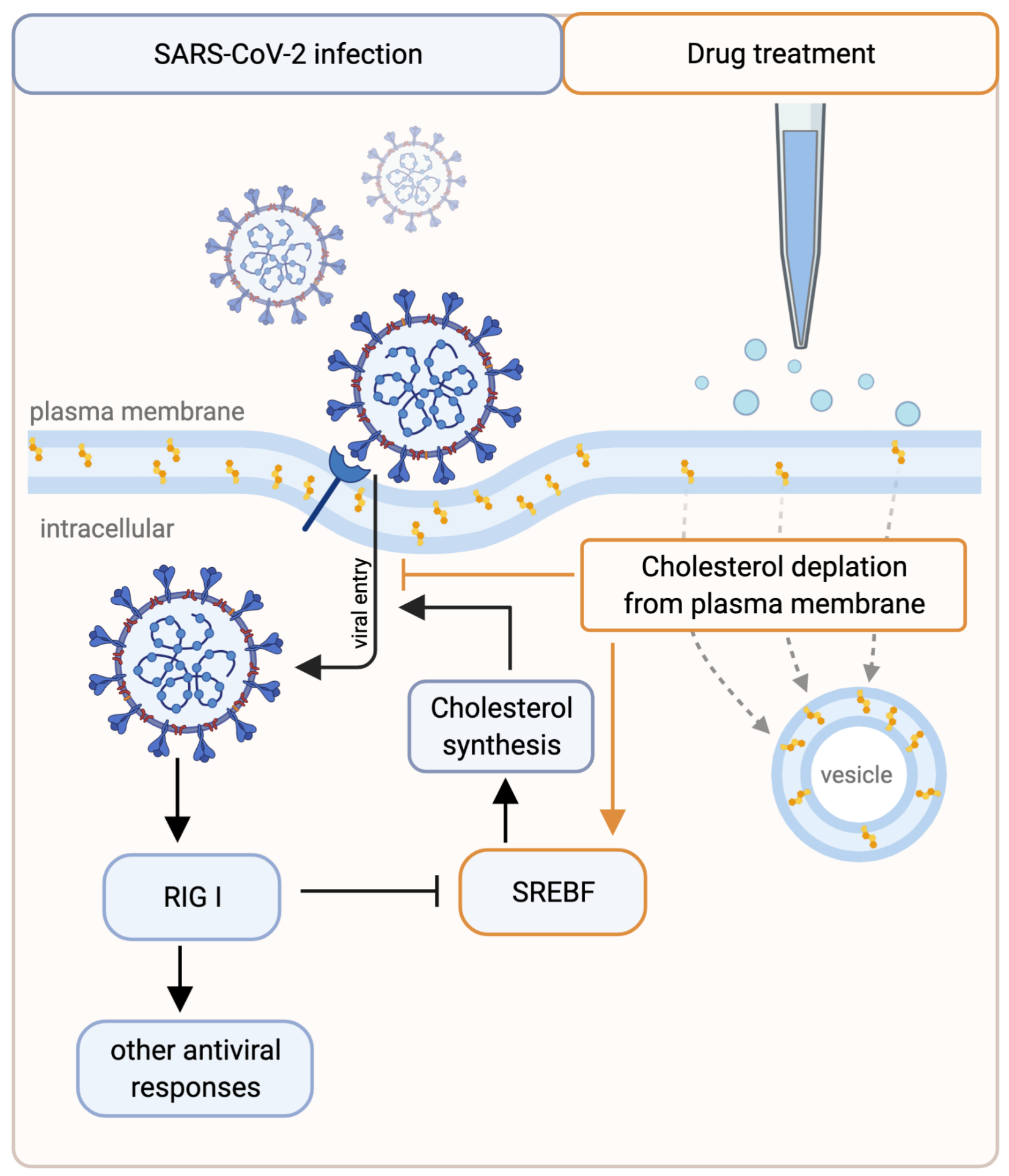




\section{Introduction}

The newly emerged Severe Acute Respiratory Syndrome Coronavirus 2 (SARS-CoV-2), causing the coronavirus disease 2019 (COVID-19), has led to more than 222,000,000 infections and $4,600,000$ deaths worldwide (Dong, Du, and Gardner 2020). Identification of new therapeutic compounds against SARS-CoV-2 / COVID-19 is an urgent need until effective vaccination is worldwide available and given the emergence of SARS-CoV-2 strains showing immune evasion (McCallum et al. 2021). The main therapeutic strategies include A) inhibiting key viral enzymes (like remdesivir (M. Wang et al. 2020)); B) modulating the infected cells to decrease viral replication (Gordon et al. 2020; Bouhaddou et al. 2020) and C) modulating the over-activation of the immune system to treat late complications like "cytokine storm" (Cuccarese et al. 2020; Ragab et al. 2020; Olbei et al. 2021). Repurposing already approved drugs for these indications is especially important as it allows a shorter time of approval for anti-SARS-CoV-2 treatment.

Comparing gene expression signatures of drugs and diseases have been previously shown to be an effective strategy to repurpose drugs for new therapeutic indications (Sirota et al. 2011). The general principle of these studies is that a drug inducing an opposite gene expression signature to a disease signature can reverse the disease-related gene expression changes, thus the disease phenotype. This "signature reversal" principle has also been used to predict effective drugs against SARS-CoV-2 infection (Napolitano et al. 2020; Zhou et al. 2020; Hoagland et al. 2020). However, these predictions lack, in most cases, mechanistic insight and experimental validation. Moreover, as infected cells activate adaptive antiviral pathways (like interferon pathway), inhibiting these pathways does not necessarily decrease viral replication.

In this study, we analysed transcriptomics data from in vitro SARS-CoV-2 infected cell lines and from cell lines treated with drugs showing anti-SARS-CoV-2 activity (effective drugs). Functional genomic analysis revealed shared transcription factor and pathway activity changes (eg. increased activity NFkB and JAK-STAT pathways) in the infected and effective drug-treated cell lines. Similarity between infection signature and drug signature was predictive for in vitro effective drugs, contradictory to the classical "signature reversal" principle. Machine learning-based prediction of effective drugs identified SREBF1 and SREBF2 transcription factors, key regulators of lipid metabolism, as important factors of antiviral drug effect. Using a fluorescently labeled cholesterol sensor, we showed the decreased level of plasma-membrane 
cholesterol in cells treated with effective drugs, like chlorpromazine, confirming the effect of these drugs on cholesterol metabolism. We also identified amiodarone, a drug decreasing plasma-membrane cholesterol content, thus a potential in vitro effective drug. Using an in vitro SARS-CoV-2 infection assay, we demonstrated that the antiviral effect of amiodarone can be reversed by cholesterol supplement, underlying the relevance of decreased plasma-membrane cholesterol in the antiviral drug effect.

\section{Results}

\subsection{Analysis of host pathway and transcription factor activities reveals adaptive response of SARS-CoV-2 infected cells}

We analysed gene expression data from two recent studies (GSE147507 (Blanco-Melo et al. 2020) and GSE148729 (Wyler et al. 2021)), where lung epithelial cancer cell lines (Calu-3 and A549) were infected with SARS-CoV-2. To identify infection-induced pathway and transcription factor (TF) changes, we used the PROGENy (Schubert et al. 2018; Holland, Szalai, and Saez-Rodriguez 2019) and DoRothEA (Garcia-Alonso et al. 2018, 2019) tools, respectively (more details in Methods).

PROGENy analysis showed increased activity of NFkB and TNFa pathways in both analysed cell lines, while the activity of JAK-STAT pathway increased more pronounced in infected Calu-3 cell lines (Figure 1A). DoRothEA analysis (Figure 1B) revealed strong activation of STAT, IRF and NFkB transcription factors, while cell growth-related transcription factors (E2Fs, Myc) showed decreased activity. Also SREBF1/2, key transcriptional regulators of cholesterol synthesis, showed decreased activity. STATs, IRFs and NFkB pathways / TFs play a key role in antiviral innate immunity (Seth, Sun, and Chen 2006). Decreased activity of E2Fs and Myc (Ramana et al. 2000) and decreased synthesis of cholesterol (York et al. 2015) are also part of the physiological antiviral / interferon response.

To further analyse which upstream signalling pathways regulate the inferred TF activity changes, we used CARNIVAL (Liu et al. 2019), a signaling network contextualisation tool, which connects transcription factor activities to perturbations in signaling networks via integer linear programming (more details in Methods). We performed CARNIVAL analysis using inferred transcription factor activities from a SARS-CoV-2 infected cell line (GSE147507, Calu-3), and 
used RIG-I like receptors (DDX58 and IFIH1), key receptors for foreign RNA sensing (Rehwinkel and Gack 2020), as main perturbation target. CARNIVAL results showed (Figure 1C), that activation of RIG-I like receptors by the dsRNA of SARS-CoV-2 can directly lead to the observed transcription factor activity changes, including activation of NFkB, IRFs and STATs and inhibition of SREBF2 and E2F4. Key identified intermediate nodes AKT1 and MAPK1 were already connected to coronavirus infection (Kindrachuk et al. 2015; Bouhaddou et al. 2020), also suggesting that the observed TF changes are initiated by the RIG-I like receptors, thus corresponding to the antiviral response of the host cell.

In summary, our functional analysis of the gene expression changes in SARS-CoV-2 infected cell lines suggests that a large part of the induced pathway / transcription factor activity changes are adaptive, i.e. part of the physiological antiviral response. 


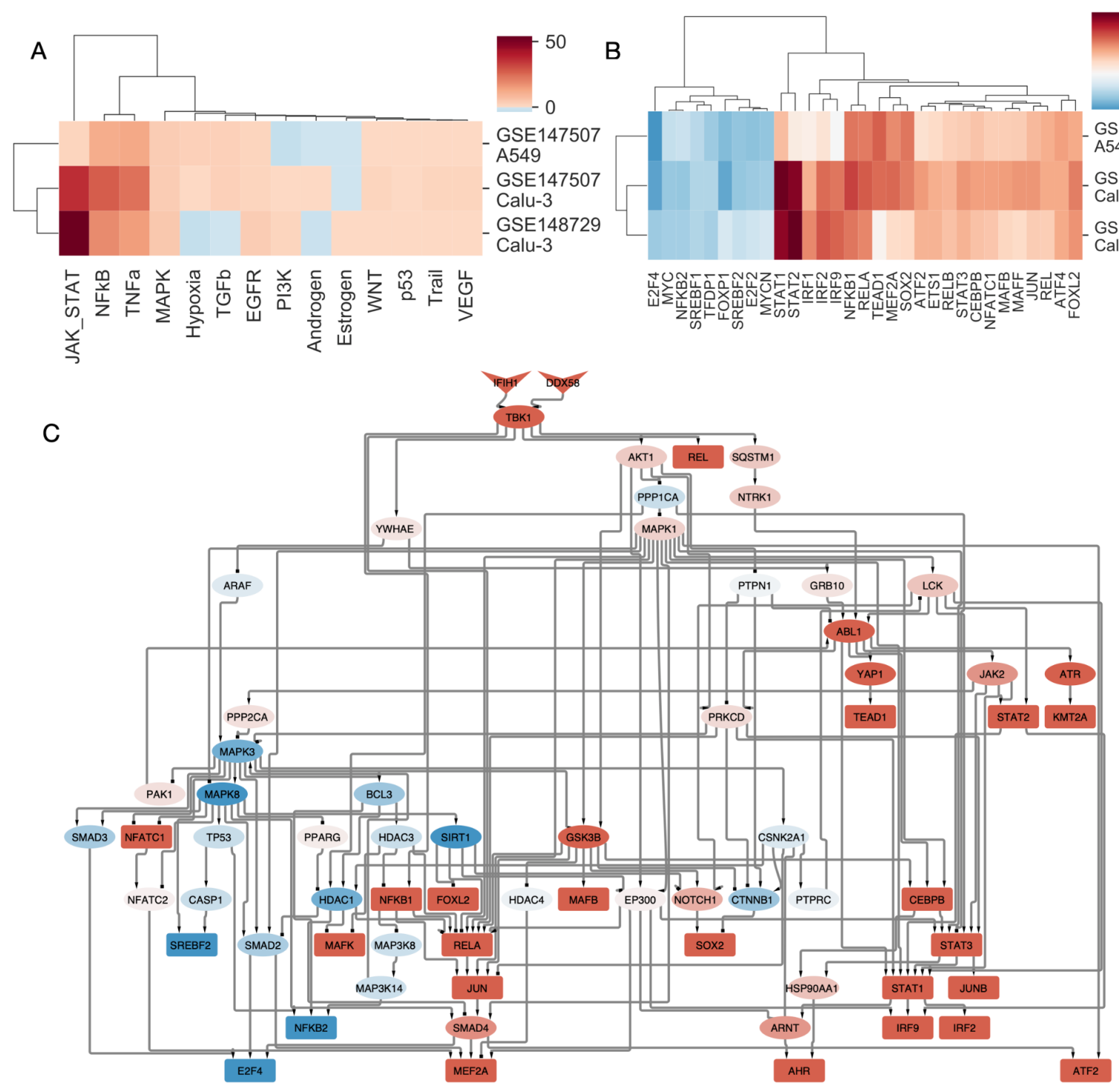

Figure 1 - Pathway and transcription factor activity analysis of SARS-CoV-2 infected cell lines

(A) Inferred pathway and (B) TF activities of SARS-CoV-2 infected samples from lung epithelial cell lines (Calu-3 and A549). Activities were calculated from differential expression signatures (infected - control) using PROGENy and DoRothEA tools for pathway and TF activities, respectively. Only TFs with high absolute level of activity changes (absolute normalised enrichment score > 4) are shown. (C) Causal signalling network in SARS-CoV-2 infected Calu-3 cells (GSE147507) identified by CARNIVAL. RIG-I like receptors (DDX58 and IFIH1) as perturbation targets and DoRothEA inferred TF activities were used as the input of the CARNIVAL pipeline. Color code represents inferred activity of protein nodes (blue: inhibited, red: activated). 


\subsection{Analysis of in vitro anti-SARS-CoV-2 drug-induced pathway and transcription factor activities reveals similar changes to virus infection}

To compare infection and drug-induced signatures, we used a large compendium of drug-induced gene expression signatures from the LINCS-L1000 project (Subramanian et al. 2017). LINCS-L1000 contains drug-induced gene expression signatures from different cell lines, concentrations and time points. We calculated consensus gene signatures for each drug using our previous approach ((Szalai et al. 2019), Methods), ending up with gene expression signatures for 4671 drugs. To select drugs effectively inhibiting SARS-CoV-2 replication in vitro, we used a curated database created by ChEMBL (http://chembl.blogspot.com/2020/05/chembl27-sars-cov-2-release.html). This dataset contains 133 drugs previously showing effective inhibition of viral replication in 8 studies (Gordon et al. 2020; Riva et al. 2020; Weston et al. 2020; Heiser et al. 2020; Si et al. 2020; Touret et al. 2020; Ellinger et al. 2021; Jeon et al., n.d.). We found an intersection of 47 drugs between LINCS-L1000 (available gene expression signatures) and ChEMBL dataset (in vitro effective drugs). To characterize drug-induced pathway and transcription activity changes, we analysed consensus drug signatures using PROGENy and DoRothEA.

PROGENy analysis showed strong activation of NFkB and TNFa pathways by several drugs, including niclosamide, perhexiline and digoxin (Figure 2A). Several drugs also strongly activated the JAK-STAT pathway (RTK inhibitors osimertinib and regorafenib). In case of TF analysis, we found similar patterns (Figure 2B) to the infection-induced signatures: increased activity of NFkB and STAT transcription factors and decreased activity of Myc/E2Fs transcription factors. Interestingly, SREBF1/2 showed strongly increased activity for a large cluster of drugs, but (similar to the infection signatures) decreased in another cluster. To further analyse the TF activity changes in the different clusters of drugs, we calculated average TF activities for these clusters and plotted these values against the average TF activities of the 3 SARS-CoV-2 infection signatures (Figure 2C). One cluster (Figure 2C, upper left panel), showed high correlation (Spearman's rho $=0.64, \mathrm{p}=8.55 \mathrm{e}-35$ ) across all TFs. Two other clusters (Figure 2C, upper middle and upper right panels) showed lower, but still significant correlation with infection TF activity signature (Spearman's rho $=0.14$ and $0.18, p=0.0122$ and 0.00174 , respectively), with prominent increase of STATs and decrease of E2F4 transcription factor activity. For the remaining two large clusters, we found either negligible (Figure $2 \mathrm{C}$, lower right panel) or high 
(Figure 2C, lower left panel) correlation with infection-induced TF activities (Spearman rho = 0.04 and $0.58, p=0.484$ and $3.14 \mathrm{e}-27$, respectively), but we found high drug induced activity of SREBF1/2 transcription factors in these clusters, opposite to the inhibition of these TFs by SARS-CoV-2 infection.

As we found that, for several drug clusters, drug-induced TF activities showed positive correlation with SARS-CoV-2 induced TF activities, we were interested in the general similarity of drug and infection-induced gene expression signatures. To achieve this we calculated the signature similarity (Spearman's correlation coefficient, which has been previously shown to be an effective metrics to analyse signature similarity for the LINCS-L1000 data (Subramanian et al. 2017; Szalai et al. 2019)) between all the 4,671 drug signatures from our LINCS-L1000 dataset and the infection signatures. We found that effective anti-SARS-CoV-2 drugs (ChEMBL dataset) have higher similarity to infection signatures, than ineffective drugs / drugs with unknown efficacy (Figure 2E, Mann-Whitney $U$ test $p$ value $=<1 e-200$ ).

In summary, we found that known in vitro effective anti-SARS-CoV-2 drugs induce similar pathway and TF activity patterns, and appropriately similar gene expression signatures to virus infection signatures. We also identified two large clusters of drugs inducing strong activation of SREBF1/2 transcription factors, key regulators of cholesterol / lipid metabolism. 


\section{A}

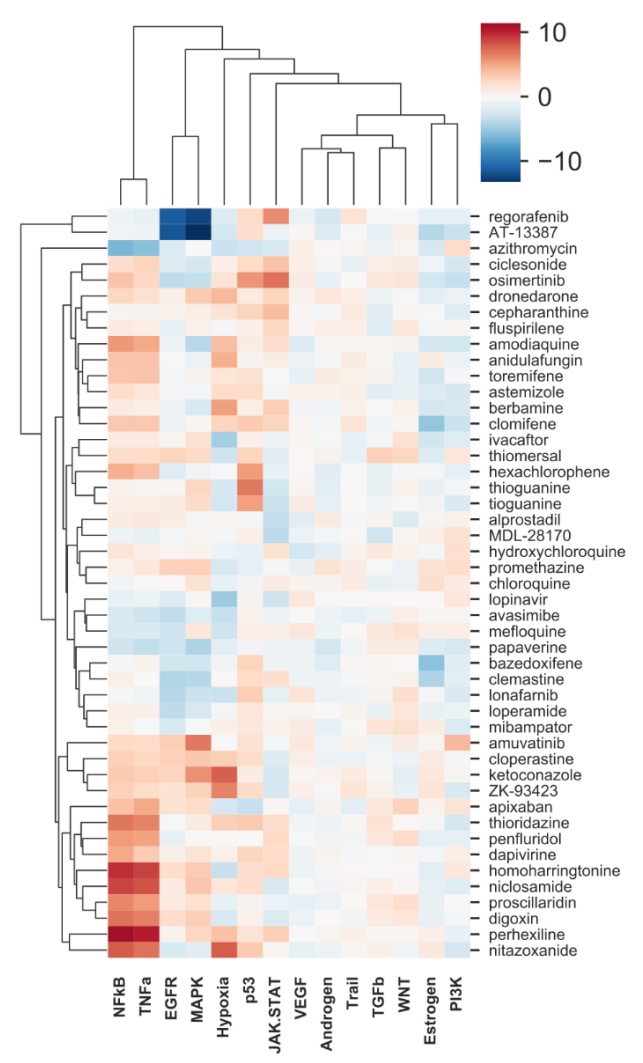

C

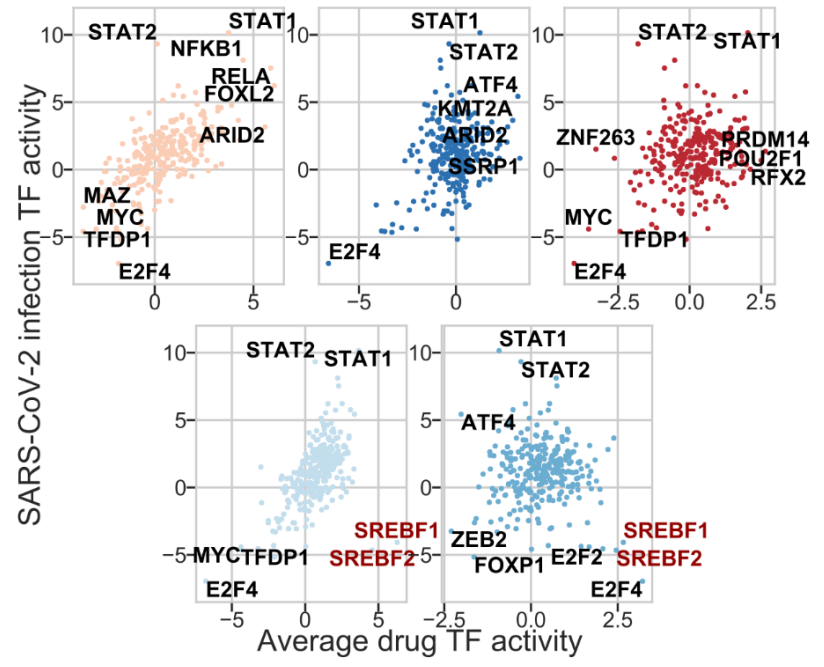

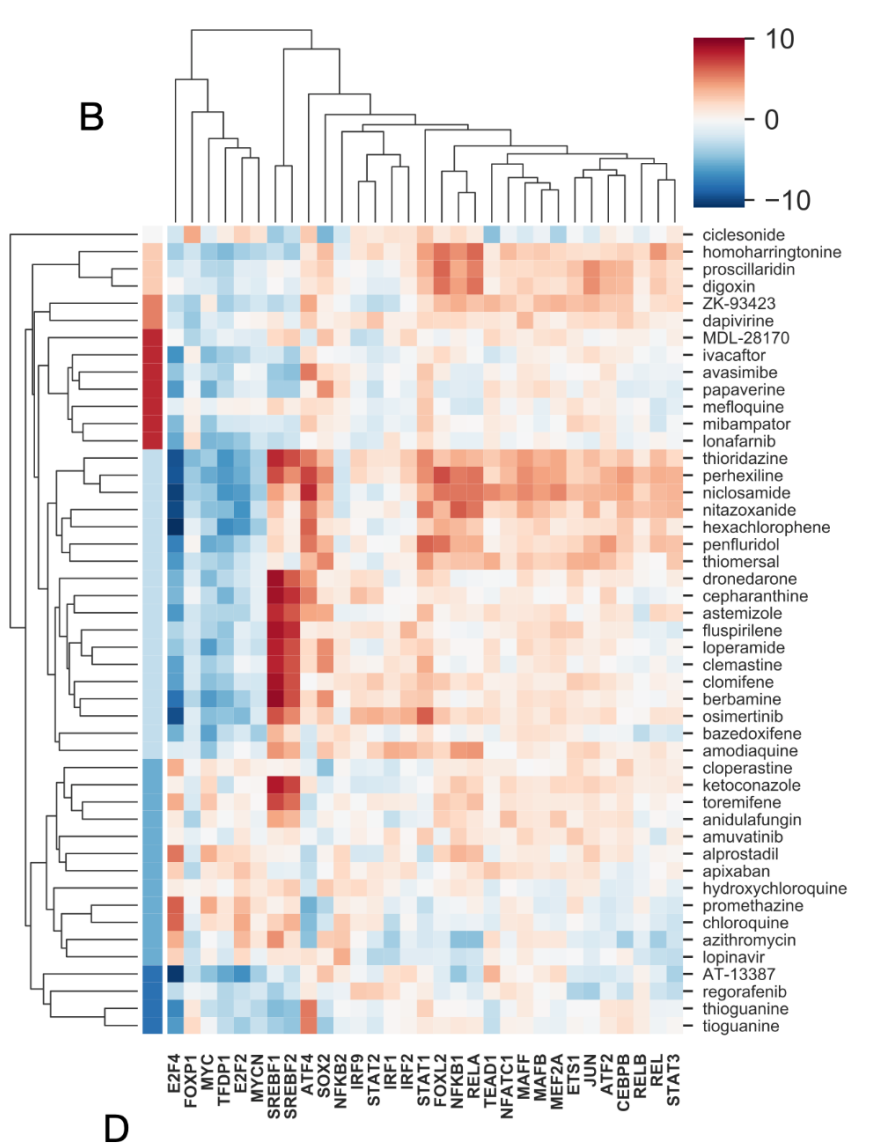

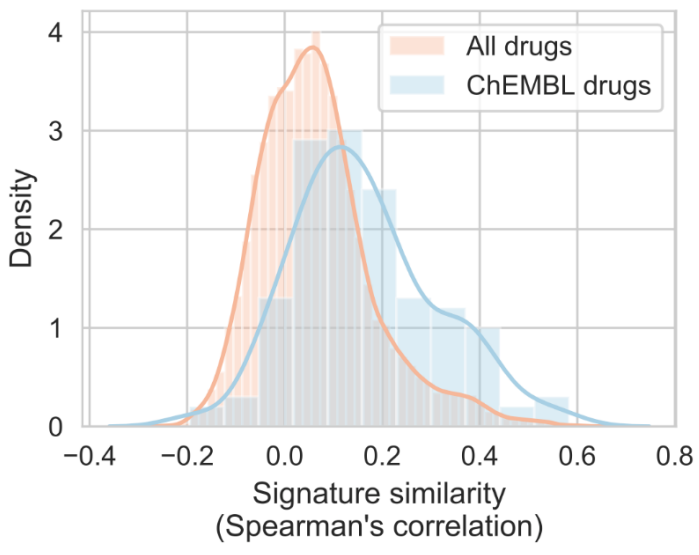

Figure 2 - Pathway and transcription factor activity analysis of effective drugs treated cell lines

(A) Inferred pathway and (B) TF activities of anti-SARS-CoV-2 drug treated cell lines. Activities were calculated from LINCS-L1000 consensus drug signatures, using PROGENy and DoRothEA tools for pathway and TF activities, respectively. Drug clusters in (B) are color coded. (C) Relationship between average TF activities induced by drug treatment and SARS-CoV-2 infection for 5 different drug clusters (colors of clusters correspond to panel B). TFs with the highest/lowest average activities are text labeled. 
(D) Density plot of similarities between SARS-CoV-2- and drug-induced signatures for all LINCS-L1000 drugs and known anti-SARS-CoV-2 drugs (ChEMBL drugs).

\subsection{Prediction of drugs with in vitro anti-SARS-CoV-2 activity}

After identifying some general patterns in the gene expression signatures of in vitro effective anti-SARS-CoV-2 drugs, we investigated how well we can predict drug effectiveness using gene expression signatures.

As a first strategy, we simply used the previously calculated drug - infection signature similarity to predict effective drugs. Using these similarity values (predicted score) and the known in vitro effective drugs (ChEMBL dataset, true positive values) we performed ROC analysis (Figure 3A). We found that similarity to infection signatures is predictive for effective drugs, i.e. drugs with high similarity to infection signature are more frequently effective (ROC AUCs: 0.75, 0.74 and 0.64 for GSE147507 A549, GSE147507 Calu-3 and GSE148729 Calu-3, respectively). To test the specificity of this signature similarity-based approach for SARS-CoV-2 infection signature, we included several other virus infection-induced gene expression signatures for SARS-CoV (GSE33267 (Sims et al. 2013), GSE148729), MERS (GSE45042 (Josset et al. 2013), GSE56677 (Selinger et al. 2014)), respiratory syncytial virus (RSV, GSE147507), influenza (GSE28166 (Li et al. 2011), GSE37571) and human parainfluenza (HPIV, GSE147507) infected Calu-3 and/or A549 cell lines. Similarity to these infection signatures showed lower predictive performance for anti-SARS-CoV-2 drugs (ROC AUC values <0.7 except one SARS and RSV signature with ROC AUCs 0.70 and 0.71 , respectively, Figure 3B), suggesting the relative SARS-CoV-2 specificity of the similarity-based methods.

Following this unsupervised prediction strategy, we also performed supervised, machine learning-based predictions. We used the drug-induced TF activities as features, and effective drugs from the ChEMBL dataset as positive examples, with Random Forest Classification as prediction algorithm. We set up a random subsampling based cross-validation scheme and evaluated the performance using ROC analysis (Methods). Our results showed a slightly improved performance compared to the unsupervised, similarity-based approach (mean ROC AUCs: 0.69 and $0.68,0.66,0.57$, respectively for the machine learning and similarity based methods, paired t-test $p$-values between machine learning and similarity based methods: 1.85e-01, 1.29e-06, 1.75e-34 for GSE147507 A549, Calu-3 and GSE148729 Calu-3 signatures 
respectively, Figure $3 \mathrm{C}$ ). To gain some more mechanistic insight from the prediction of machine learning models, we analysed feature importances (Gini importance, Figure 3D) of the Random Forest Regression models and found that SREBF1 and SREBF2 activity were the two most important features, followed by HNF4A, TFAP2A and TP63 transcription factors.

In summary, our two different prediction approaches showed reasonable performance to predict drugs with in vitro anti-SARS-CoV-2 activity, and also highlighted the importance of previously discussed SREBF1/2 transcription factors. Drug - SARS-CoV-2 signature similarities, and predicted probabilities of anti-SARS-CoV-2 activity is available in Supplementary Table 1.
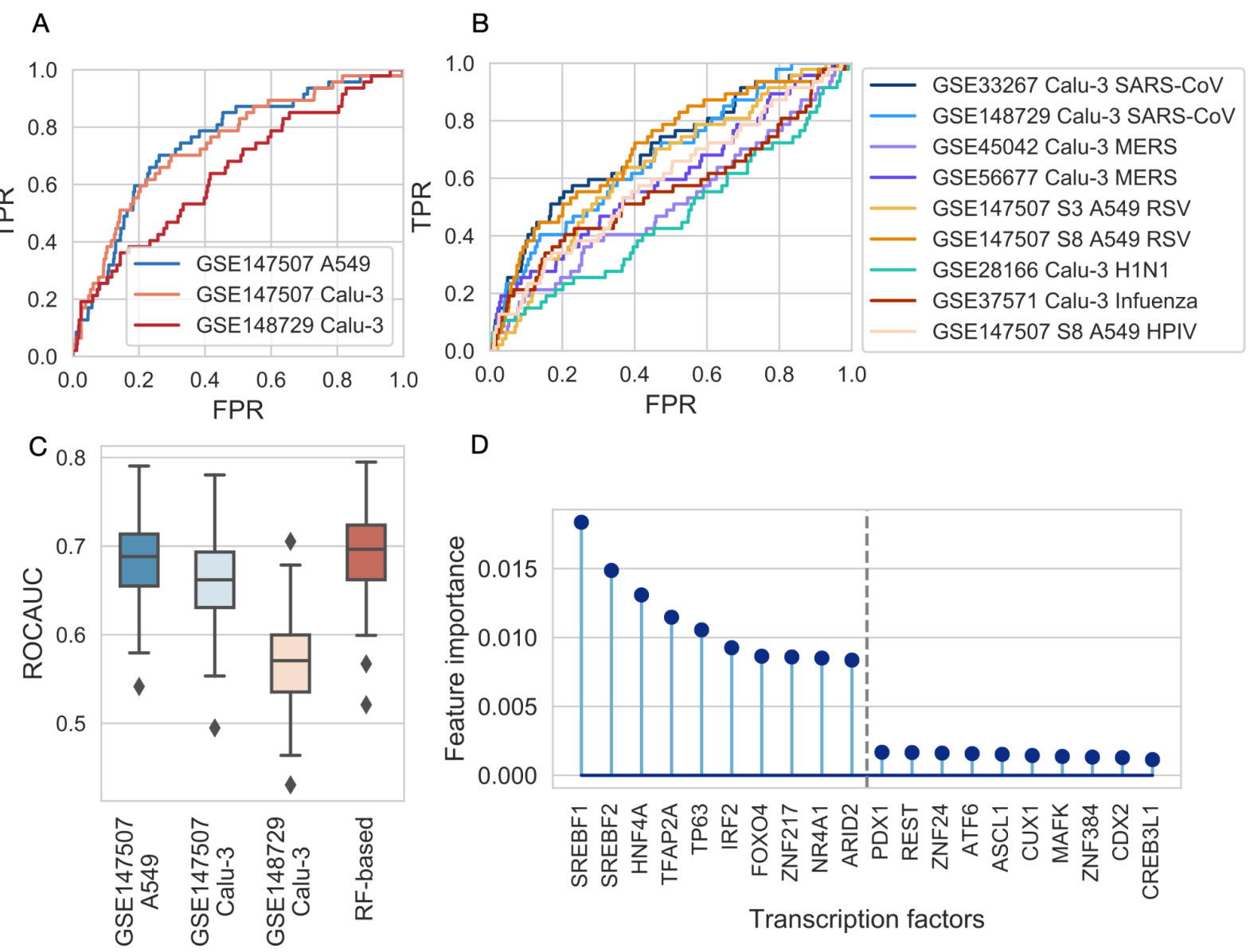

D

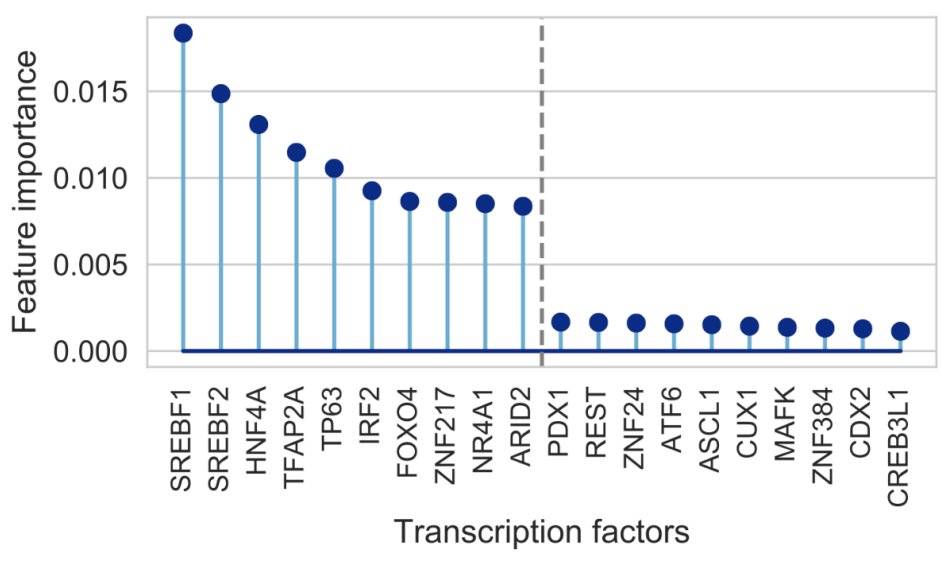

Figure 3 - Evaluation of similarity-based and machine learning-based models in predicting in vitro effective drugs

$(A, B) R O C$ analysis of similarity-based predictions of effective drugs against SARS-CoV-2. Drug SARS-CoV-2 (A) or drug - other virus infection signature similarity was used as prediction score, while known in vitro effective drugs (ChEMBL dataset) were used as true positives. (FPR: false positive rate, 
TPR: true positive rate) (C) Comparison of predictive performance (ROCAUCs) of similarity-based method (similarity to SARS-CoV-2 infection signature, $x$-axis) and random forest-based (RF-based, $x$-axis) prediction. Results of 100 random subsampling cross-validations. In case of similarity based methods, ROC AUC curves were only calculated for the corresponding cross-validation sets. (D) Feature importances (Gini importance) of the Random Forest model. Top and bottom 10 features (TFs) are shown according to importance.

\subsection{Anti-SARS-CoV-2 drugs are increasing SREBF activity by depleting plasma membrane cholesterol}

While in most cases we found similarity between the activity of SARS-CoV-2 infection and in vitro effective drug induced transcription factor activities, in case of SREBF1/2 we found opposite changes: SARS-CoV-2 infection inhibited SREBF1/2, while a large cluster of effective drugs lead to increased activity of SREBFs. SREBFs are activated through the decreased cholesterol content of plasma membrane and endoplasmic reticulum, and activated SREBFs induce the expression of cholesterol, and other lipid synthesizing enzymes (Horton, Goldstein, and Brown 2002). From this point of view, decreased SREBF activity during viral infection can lead to decreased cholesterol synthesis, which can inhibit the viral replication and/or viral entry (York et al. 2015), thus can be considered as an adaptive response of the host cell (Figure 4A). Interestingly, we observed a strongly increased SREBF activity in large clusters of effective drugs. To resolve this discordance, we hypothesized that these in vitro effective drugs directly decrease plasma membrane cholesterol (Figure 4A). In this case, drug induced decrease of plasma membrane cholesterol can contribute to the antiviral effect, while decreased cholesterol levels can activate SREBFs, thus explaining the observed increased activity of these TFs in our bioinformatic analysis.

To confirm this hypothesis, we performed high-throughput, automatic confocal microscopy imaging using a fluorescently labeled cholesterol sensor domain, D4H-mVenus (Maekawa and Fairn 2015; Maekawa 2017). HEK293A cells were co-transfected with D4H-mVenus and cytoplasmic Cerulean as cytosolic marker (Figure 4B), and treated with dimethyl sulfoxide (DMSO, negative control), M $\beta C D$ (methyl $\beta$-cyclodextrin, plasma membrane cholesterol depleting compound, as positive control) and 3 drugs from our computational drug repurposing pipeline, loperamide, amiodarone and chlorpromazine (all drugs were used in $10 \mu \mathrm{M}$ final concentration). All these three drugs increased the activity of SREBF transcription factors 
(Figure 4B, left panel). Loperamide and chlorpromazine have been previously shown to be in vitro effective against SARS-CoV-2 (ChEMBL dataset), while amiodarone was one of the top predicted drugs of the Random Forest model (Figure 4B, right panel, ranked 36/4671 drugs, Supplementary Table 1). We also treated HEK293A cells with rosuvastatin, an inhibitor of cholesterol synthesis. Rosuvastatin also alters cellular cholesterol metabolism, however, it does not influence plasma membrane cholesterol directly, but inhibits HMG-CoA reductase, the rate limiting enzyme of de novo cholesterol synthesis. Rosuvastatin was not predicted as an effective anti-SARS-CoV-2 drug by the Random Forest model (Figure 4B left panel, ranked 1821/4671 drugs).

Cells were treated with the different drugs and serial confocal microscopy images were recorded for 4.5 hours. In untreated, or DMSO treated cells, we observed a predominantly plasma membrane localisation of the fluorescent protein labeled cholesterol sensor (Figure 4D, top left panel). Treatment with $\mathrm{M} \beta C D$ led to decreased plasma membrane cholesterol levels, while cholesterol accumulated in intracellular vesicles (Figure 4D, top right panel). We observed similar phenotypic changes in case of amiodarone and chlorpromazine (Figure 4D, bottom panels), while the localisation of cholesterol sensor in loperamide and rosuvastatin treated cells was more similar to control condition (Supplementary Figure 1).

For a more systematic and unbiased analysis of the changes in the localisation of cholesterol sensors, we performed quantitative image analysis (Supplementary Figure 2). For each cell in each image, we calculated the ratio of average plasma membrane (PM) and average intracellular (IC) D4H-mVenus fluorescence (PM/IC ratio). To segment cells in confocal microscopy images, we used Cellpose library ((Stringer et al. 2021), Methods). Plotting the $\mathrm{PM} / \mathrm{IC}$ ratio as a function of elapsed time after drug treatment (Figure 4E) revealed that PM/IC ratio did not decrease in loperamide and rosuvastatin treated samples, while $M \beta C D$, chlorpromazine and amiodarone treatment induced significant decrease of the ratio (linear model coefficients values for interaction between drug treatment and time: $-0.002,-0.00086$, $-0.00017,-0.000032$ and 0.000083 for M $\mathrm{BCD}$, chlorpromazine, amiodarone, loperamide, rosuvastatin respectively, $p$ values: $<1 \mathrm{e}-200$, $<1 \mathrm{e}-200,2.71 \mathrm{e}-09,0.25$ and 0.0047 ), confirming the plasma membrane cholesterol depleting effect of chlorpromazine and amiodarone, two SREBF activating drugs. 
bioRxiv preprint doi: https://doi.org/10.1101/2021.09.10.459786; this version posted September 10, 2021. The copyright holder for this preprint (which was not certified by peer review) is the author/funder, who has granted bioRxiv a license to display the preprint in perpetuity. It is made available under aCC-BY 4.0 International license.

In summary, our high-throughput image acquisition and analysis pipeline confirmed that chlorpromazine and amiodarone decreased plasma membrane cholesterol content, which explains the increased activity of SREBF transcription factors in case of gene expression readout.

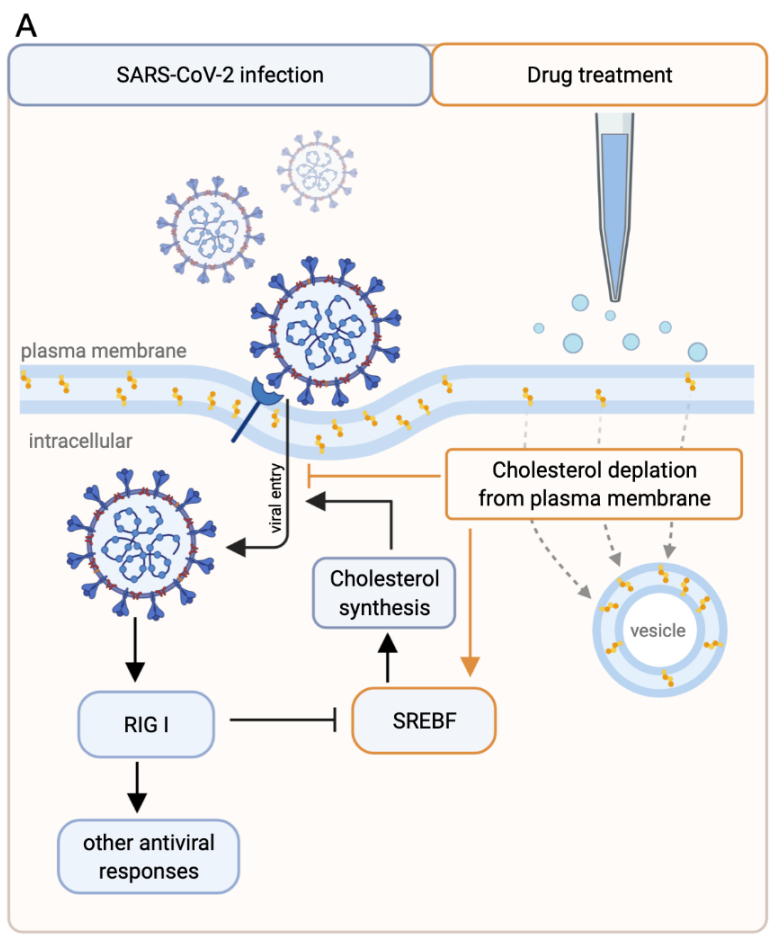

B

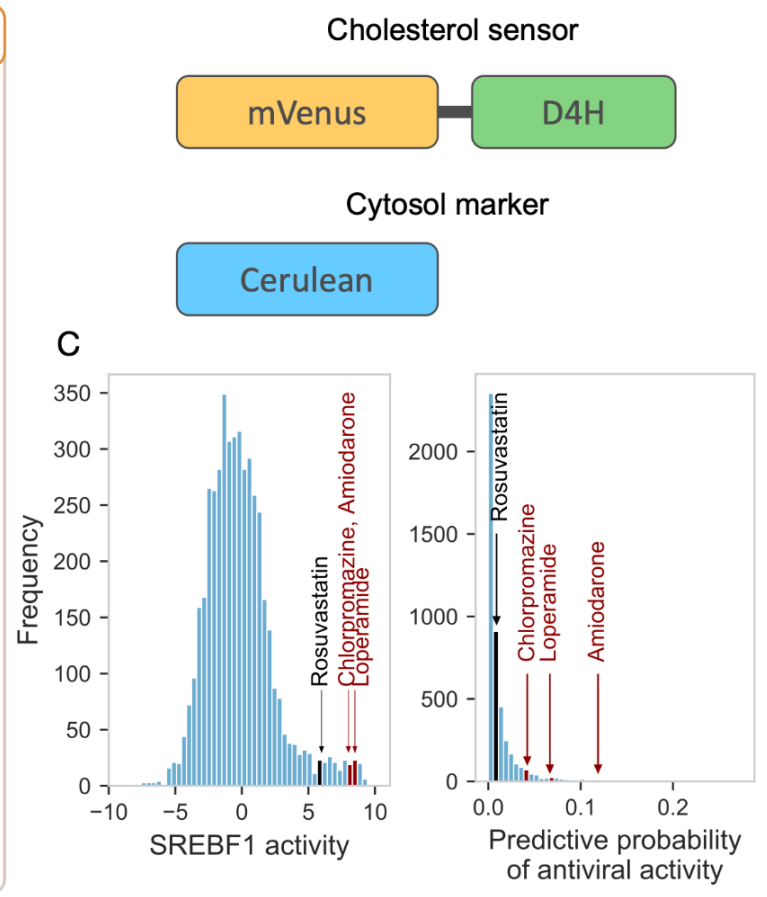

D DMSO

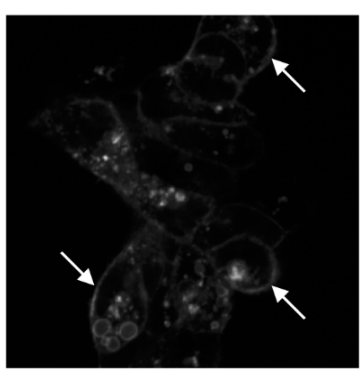

Chlorpromazine

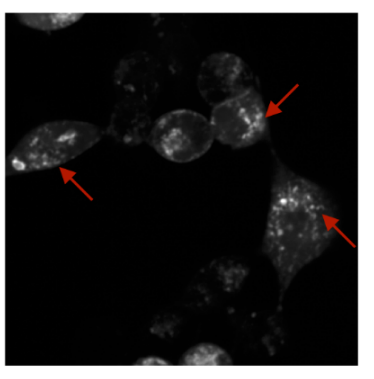

MBCD

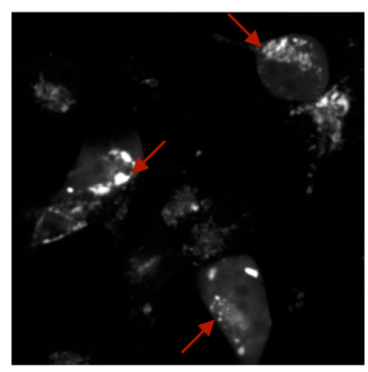

Amiodarone

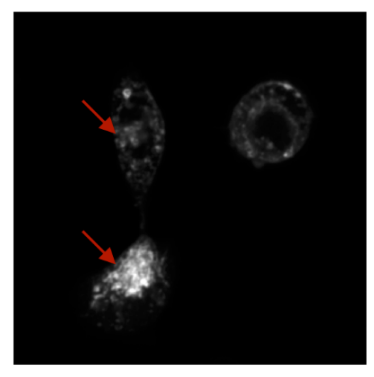

E

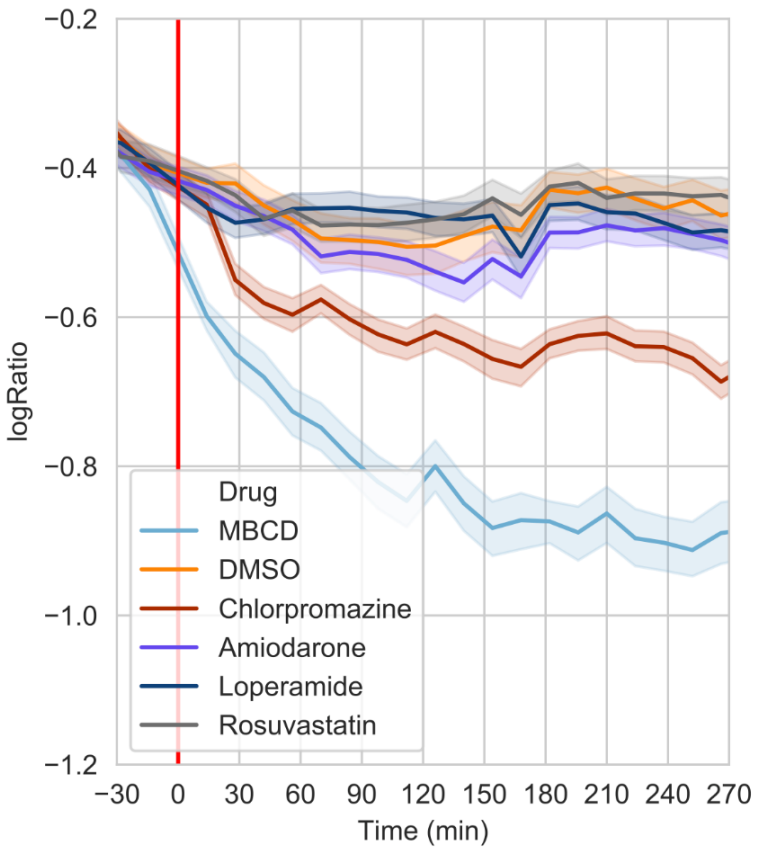




\section{Figure 4 - Cholesterol depleting effect of SREBF activating drugs}

(A) Schematic figure of the hypothesis that antiviral drugs block virus entry into cells by cholesterol depletion from plasma membrane, and are leading to a compensatory increased SREBF1/2 activity. (B) Schematic representation of the used fluorescent constructs. (C) Histogram of SREBF1 activation (left panel) and histogram of predicted probabilities of in vitro antiviral activity of LINCS-L1000 drugs (right panel). Drugs selected for in vitro experiments are text labeled. (D) Representative confocal microscopy images of D4H-mVenus transfected HEK293A cells treated with DMSO, M $B C D$, chlorpromazine or amiodarone. White arrows mark plasma membrane, while red arrows show intracellular localised cholesterol sensors. (E) Time-dependent change of $\log _{2}(P M / I C$ ratio) of average cholesterol sensor intensity in HEK293A cells treated with DMSO, MBCD, chlorpromazine, amiodarone, loperamide or rosuvastatin. Red line marks drug treatment.

\subsection{Cholesterol rescue inhibits the anti-SARS-CoV-2 activity of amiodarone}

As our experiments revealed that the selected drugs with in vitro anti-SARS-CoV-2 activity decreased the cholesterol content of plasma membrane, we were interested in whether decreased plasma membrane cholesterol levels could play a causal role in the antiviral effect, according to our assumptions (Figure 4A). To test this hypothesis, we performed in vitro SARS-CoV-2 viral infection assay with cholesterol rescue in Vero-E6 cells.

At first we tested whether the investigated drugs show anti-SARS-CoV-2 activity in our previously described experimental system (Konrat et al. 2020). Briefly, Vero-E6 cells were co-treated with SARS-CoV-2 and the selected drugs (6 $\mu \mathrm{M}$ amiodarone, $12 \mu \mathrm{M}$ chlorpromazine or $50 \mu \mathrm{M}$ loperamide, effective drug concentrations were selected based on preliminary experiments) for 30 minutes, then washed and incubated with the drugs for 48 hours. Infection efficacy was evaluated by microscopic examination of infection induced cytopathic effect (CPE, more details in Methods). Untreated, SARS-CoV-2 infected cells showed strong cytopathy (Figure 5A, top left panel), while amiodarone, chlorpromazine and loperamide markedly reduced the infection induced cytopathy, confirming the antiviral effect of these drugs (Figure $5 \mathrm{~A})$. The used compounds did not lead to cellular toxicity in the used concentrations (Supplementary Figure 3).

To test the effect of plasma membrane cholesterol depletion on SARS-CoV-2 infectivity, we performed cholesterol rescue experiments (Figure 5B). Vero-E6 cells were treated with drugs 
overnight, then the media was replaced with cholesterol $(80 \mu \mathrm{M})$ containing media. After 1 hour of cholesterol treatment, the cells were infected for $30 \mathrm{~min}$ with SARS-CoV-2. Infection efficacy was evaluated 48 hours after infection by droplet digital PCR based viral RNA quantification (Figure 5C). Chlorpromazine and loperamide did not have antiviral effect in the pretreatment setting (linear model p values: 0.62 and 0.18 , respectively), while amiodarone decreased viral particle number significantly (linear model $p$ value: 1.47e-05). Cholesterol replenishment significantly increased viral particle number in amiodarone treated Vero-E6 cells (amiodarone : cholesterol interaction term $p$ value: 0.026), confirming the causal role of drug induced cholesterol depletion in the antiviral effect of amiodarone.

Our in vitro SARS-CoV-2 infection assay confirmed the antiviral effects of chlorpromazine, loperamide and amiodarone, and cholesterol rescue experiments suggest that plasma membrane cholesterol depletion plays an important role in the antiviral effect of amiodarone. 
A

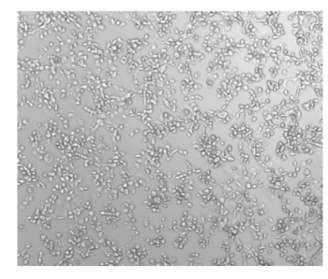

SARS-CoV-2

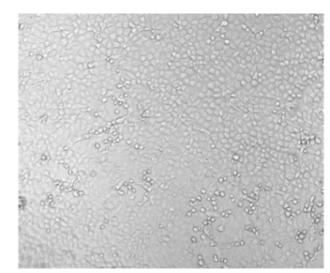

SARS-CoV-2 + Chlorpromazine $(12 \mu \mathrm{M})$

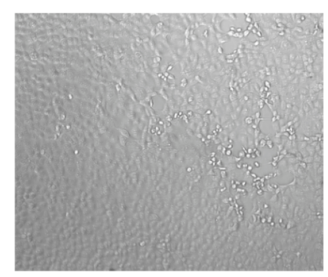

SARS-CoV-2 + Amiodarone $(6 \mu \mathrm{M})$

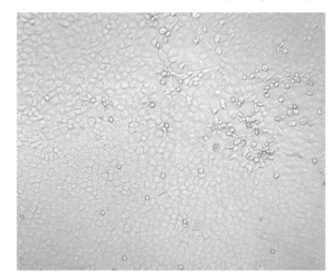

SARS-CoV-2 + Loperamide $(50 \mu \mathrm{M})$
C

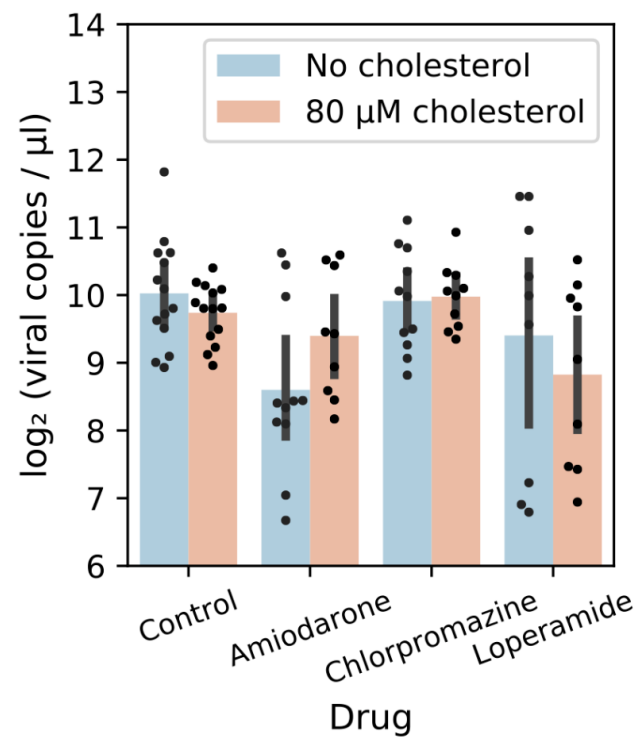

B
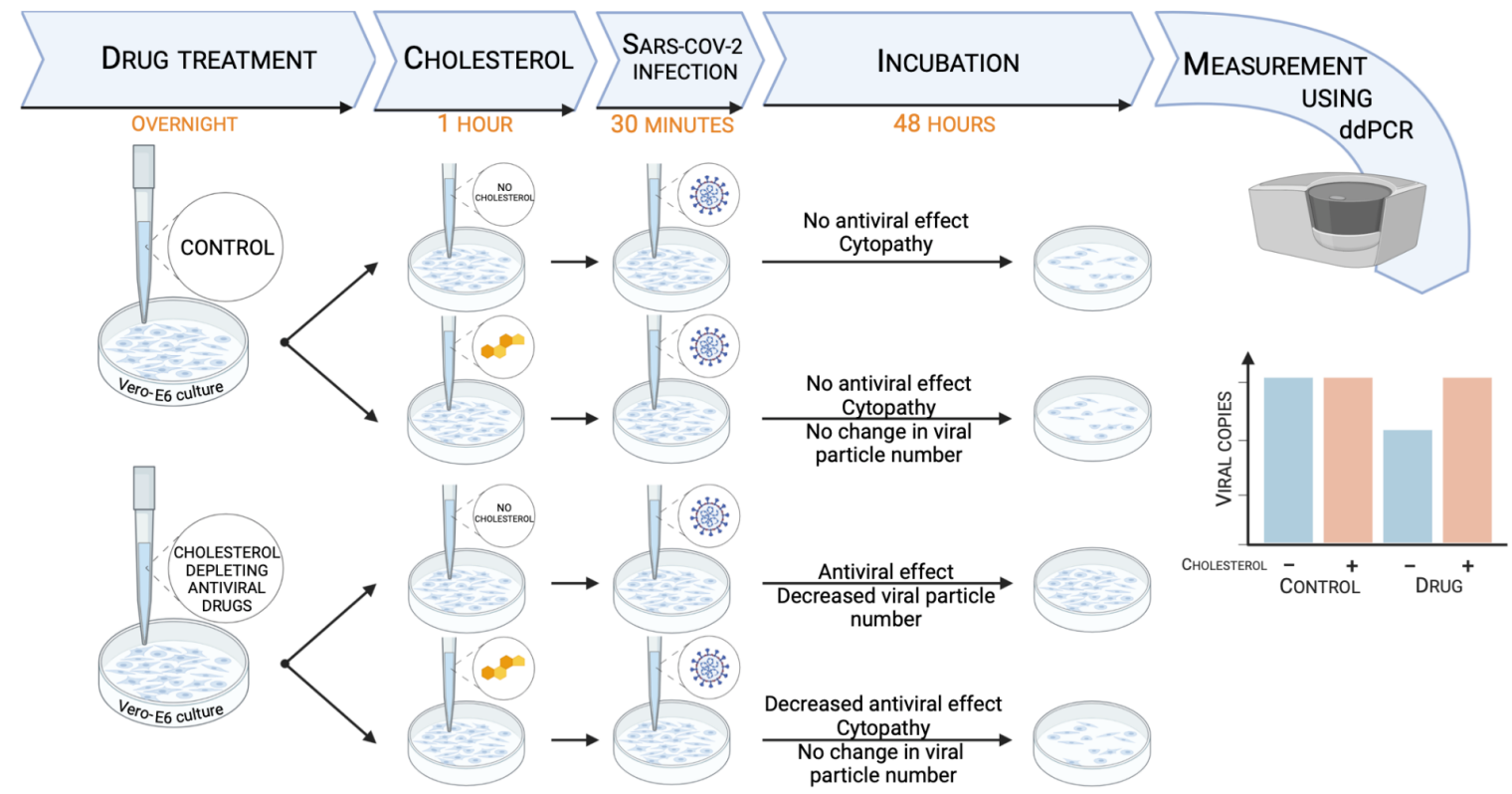

\section{Figure 5 - Cholesterol replenishment inhibits antiviral effect of amiodarone}

(A) Predicted drugs inhibit SARS-CoV-2 replication in infected Vero-E6 cells. Vero-E6 cells were infected with SARS-CoV-2 (top left) and co-treated either with amiodarone (top right), chlorpromazine (bottom left) or loperamide (bottom right). Antiviral effect (reduced cytopathy) was evaluated by microscopic imaging (10x objective) 48 hours after infection. (B) Schematic figure of cholesterol rescue experiments. (C) Effect of cholesterol rescue on antiviral drug effect. Vero-E6 cells were pretreated with drugs (x-axis), cholesterol was replenished (color code) and cells were infected with SARS-CoV-2. Antiviral effect of drugs was evaluated 48 hours after infection by droplet digital PCR (viral copies, y axis). 


\section{Discussion}

In this study, we analysed the gene expression signatures of in vitro SARS-CoV-2 infected cells and effective anti-SARS-CoV-2 drugs. Using functional genomic computational tools, we showed that both virus infection and drug treatment leads to similar changes of pathway and transcription factor activities, like activation of antiviral NFkB and JAK-STAT pathways. Signature similarity between infection and drug signature was predictive for drugs with in vitro anti-SARS-CoV-2 activity, contrary to the classical "signature reversal" hypothesis. Using machine learning models we effectively predicted anti-SARS-CoV-2 drugs, and predicted amiodarone as an in vitro antiviral compound. More detailed functional genomic analysis of TF activities revealed that SREBF1/2 TFs are strongly activated by large clusters of effective drugs. Using a high-throughput confocal microscopy setup and quantitative image analysis we showed that two of the three investigated effective drugs influence cellular distribution of cholesterol, leading to decreased plasma membrane cholesterol content. Viral infection assay confirmed the already described in vitro antiviral activity of loperamide and chlorpromazine, and also the predicted antiviral activity of amiodarone. Cholesterol supplement reversed the antiviral effect of amiodarone, suggesting the causal role of decreased membrane cholesterol in the antiviral effect.

Gene expression based computational drug repurposing is a promising field to find new disease indications of existing drugs (Pushpakom et al. 2019). Despite its simplicity, it has been used successfully to identify repurposable drugs for different diseases from cancer (B. Chen et al. 2017; Stathias et al. 2018) through inflammatory (Malcomson et al. 2016) to metabolic (Kunkel et al. 2011) diseases. While most of the related works rely on the "signature reversal" hypothesis, in case of infection diseases, like COVID-19, it is less clear whether signature reversal (inhibiting the virus-hijacked signalisation) or signature similarity (promoting the antiviral response of infected host cells) can be more effective. While early studies at the beginning of the COVID-19 pandemic applied mostly the original signature reversal hypothesis, more recent works (Laise et al. 2020; F. Chen et al. 2021) also assumed that drugs with similarity to the SARS-CoV-2 induced gene expression signature can be effective. In our work, we performed a more unbiased analysis of signature based drug repurposing against SARS-CoV-2. We compared the gene expression signatures of known effective drugs against SARS-CoV-2 
infection signatures, and found that signature similarity, and not dissimilarity, is predictive for antiviral effect. These results suggest that increasing the antiviral response of host cells can be a more effective strategy than inhibiting viral infection induced pathways. Whether this is specific for SARS-CoV-2, known for evading several antiviral systems of the host cell (Lei et al. 2020), or a general mechanism for (viral) infections, needs further analysis with large scale in vitro drug screenings against other viruses. Nevertheless, using our "signature similarity" principle instead of - or together with - the "signature reversal" hypothesis can accelerate computational drug repurposing against existing and emerging infectious diseases.

While signature (dis)similarity based computational drug repurposing has promising predictive performance, it gives no real mechanistic insight. To overcome this problem, we performed extensive functional genomic analysis of SARS-CoV-2 and drug induced gene expression signatures. We found that both viral infection and effective drugs stimulate known antiviral pathways like NFkB and JAK-STAT. We observed lower induction of these pathways in virus infected A549 cells, compared to Calu-3 cell lines, probably based on the lower expression of virus receptor ACE2 in A549 cells. The activation of antiviral pathways in virus infected and effective drug treated cells also supports the "signature similarity" principle.

Beside the activation of antiviral TFs and pathways, we also observed inhibition of (inferred) SREBF1/2 transcription factors in SARS-CoV-2 infected samples, while an activation of these TFs in a large cluster of antiviral drug treated cells. SREBF1/2 regulate the expression of key members of cholesterol synthesis. Cholesterol depletion of plasma membrane can reduce SARS-CoV-2 infection (S. Wang et al. 2020; Sanders et al. 2021), and decreasing SREBFs activity (and cholesterol synthesis) can be also part of the physiological antiviral response of the host cell (York et al. 2015). In contrast, we found increased activity of SREBFs in case of several effective drug induced gene expression signatures. Previous works also showed the increased expression of lipid metabolic enzymes (Hoagland et al. 2020) in antiviral drug treated cells, and a recent large scale CRISPR screen (Daniloski et al. 2021) also found that increased cholesterol synthesis can reduce SARS-CoV-2 infection. However, these two later conclusions were based on the analysis of gene expression changes of the cholesterol synthetic pathway. Gene expression changes are in several cases not the cause, but the (compensatory) consequence of perturbed cell states (Dugourd and Saez-Rodriguez 2019; Szalai and Saez-Rodriguez 2020). Based on this, we hypothesized that increased SREBF1/2 activity (based on transcriptional 
readout) can be a compensatory consequence of decreased plasma membrane cholesterol levels in case of several antiviral drugs. Using a fluorescent cholesterol sensor, we found that amiodarone and chlorpromazine, two effective in vitro antiviral drugs, indeed decreases the cholesterol content of plasma membranes, which can explain the (compensatory) increased SREBF1/2 activity. In an in vitro SARS-CoV-2 infection assay, coupled with cholesterol rescue, we also showed that cholesterol replenishment reduced the antiviral activity of amiodarone, thus confirmed the causal role of plasma membrane cholesterol decrease in the antiviral effect of amiodarone. While our computational analysis also predicted that PM cholesterol depletion plays a role in the antiviral effect of chlorpromazine and loperamide, we were not able to verify these predictions experimentally. Noteworthy, these two drugs had antiviral effect in case of co-treatment with virus infection, but not in the case of the pre-treatment setup used in cholesterol rescue experiments (probably due to pharmacokinetic factors). It is thus hard to draw conclusions about the role of cholesterol in the antiviral effect of these drugs.

While we showed that PM cholesterol depletion can be an important factor in the in vitro antiviral effect of drugs, whether this can be translated to in vivo is still an open question. A recent large scale study (Tummino et al. 2021) showed that several in vitro repurposable drugs exert their antiviral effect via altering the membrane composition of drug treated cells, and this antiviral effect has low translation potential based on concerns regarding drug concentration and adverse effects. While the authors of this study concluded that phospholipidosis is the main drug induced membrane component change, our results argue that altered cholesterol content can also be a causal factor in the antiviral effect of drugs. Whether altered lipid composition of cellular membranes is only a factor confounding drug repurposing studies, or this effect can be exploited towards effective therapy, needs further studies.

In summary, our study showed that in vitro SARS-CoV-2 infection and effective antiviral drugs lead to similar pathway and transcription activity changes. We found that gene expression signature similarity, and not the dissimilarity, predicts in vitro effective antiviral compounds, which can accelerate computational drug repurposing against infectious diseases, and we made the results of our predictions available for the research community (Supplementary Table 1). We also identified that plasma membrane cholesterol depletion plays an important role in the mechanism of action of several antiviral drugs, and that cholesterol replenishment inhibits the in 
vitro antiviral effect of amiodarone, thus our results also give mechanistic insight about the antiviral effect of repurposable drugs.

\section{Methods}

\section{Virus infection-induced gene expression signatures}

Microarray gene expression profiles of different virus-infected cell lines were downloaded from Gene Expression Omnibus (GEO) with accession numbers GSE28166 (H5N1), GSE37571 (Influenza), GSE33267 (SARS-CoV-1), GSE56677 and GSE45042 (MERS-CoV). Preprocessing and differential expression (DE) analysis was performed by using $\mathrm{R}$ package limma (Ritchie et al. 2015).

Total RNA-Seq profiles of SARS-CoV-2 and other virus-infected human cell lines were downloaded from GEO with accession numbers GSE147507 (SARS-CoV-2, RSV, IAV, HPIV) and GSE148729 (SARS-CoV-1 and 2). Differential expression (DE) analysis was performed using R library DESeq2 (Love, Huber, and Anders 2014).

In all gene expression datasets, we used (virus infected - control) contrasts for differential expression calculation, where the control condition was mock infection. Where gene expression data after multiple time points were available, we used $24 \mathrm{~h}$ post-infection data. Shared genes across all datasets were selected and further analyzed.

\section{Drug treatment-induced signatures}

We used Level 5 gene expression profiles from the LINCS-L1000 dataset (Subramanian et al. 2017). We calculated consensus expression signatures for each drug (across different cell lines, concentrations and time points) using the MODZ method (Szalai et al. 2019; Subramanian et al. 2017). We matched LINCS-L1000 drugs with ChEMBL effective drug dataset (http://chembl.blogspot.com/2020/05/chembl27-sars-cov-2-release.html) using drug names and simplified molecular-input line-entry system (SMILES). Only measured (landmark) genes were used in the further analysis. 


\section{Pathway and transcription factor activity analysis}

From previously calculated SARS-CoV-2 infection and effective drug-induced signatures, we inferred pathway activities using PROGENy (R package progeny, (Schubert et al. 2018; Holland, Szalai, and Saez-Rodriguez 2019)) and transcription factor activities using DoRothEA (R package dorothea, (Garcia-Alonso et al. 2019)).

PROGENy was applied to infer activities of 14 different pathways from expression and weight of their footprint gene sets. Z-scores of pathway activities were calculated using 10000 permutations of genes as background distribution. DoRothEA was applied to infer transcription factor activities using the viper algorithm (Alvarez et al. 2016). DoRothEA is a collected, curated resource of signed TF-target interactions. Interactions are assigned a confidence level ranging from $A$ (highest) to $E$ (lowest) based on the number of supporting evidence. In this study interactions assigned $A, B, C$ confidence levels were used.

We used the CARNIVAL tool ((Liu et al. 2019)) to contextualize our transcriptomics-based results into a mechanistic causal network. Briefly, CARNIVAL takes as input a prior knowledge network and a set of constraints and infers the most likely causal interactions by solving an integer linear programming problem. We assembled a curated prior knowledge signaling network from OmniPath resources ((Türei et al. 2021)). As constraints, we selected the RIG-I like receptors (DDX58 and IFIH1) as upstream signaling perturbation and the top 25 most deregulated TFs (according to DoRothEA and viper results) upon SARS-CoV-2 infection as their downstream target. In addition, we used PROGENy pathway activity scores to weight the prior knowledge network and assist CARNIVAL in the discovery of optimal networks connecting the upstream perturbation (RIG-I like receptors) to the downstream targets (TFs).

\section{Signature similarity and machine learning-based prediction}

We calculated similarities using Spearman's correlation between each virus infection-induced and each drug treatment-induced signature after selecting shared genes.

TF activity scores from drug treated cells were used to predict effective drugs against SARS-CoV-2 using Random Forest Classifier from scikit-learn Python library (Pedregosa et al. 2011). The model was trained with default parameters (100 trees) and with 100 different training sets. Training sets consisted of a $50 \%$ random sampling of effective drugs and non-effective drugs as well. The average importance of features (TFs) was computed (sum of feature 
importances, divided by the number of models). Predictive probabilities of drugs were also computed in each prediction and the mean of them was calculated (probabilities were summed for each drug and divided by the number of occurrences in validation sets).

We performed ROC analysis using scikit-learn Python library to evaluate similarity-based and machine learning-based predictions. Effective drugs against SARS-CoV-2 curated by ChEMBL and overlapping with drugs of the LINCS-L1000 dataset were used as the positive class. The negative class consisted of the part of drugs from the LINCS-L1000 dataset not considered as effective by ChEMBL. To compare machine learning-based and similarity-based methods ROC curves were computed for each different validation set (100) and signature similarity scores of the corresponding drugs were considered.

\section{Fluorescent cholesterol sensor experiments}

The cellular cholesterol sensor used in this study was the $\mathrm{D} 4 \mathrm{H}$ domain ((Maekawa and Fairn 2015; Maekawa 2017)) fluorescently labeled with monomer Venus (mVenus) on its N-terminus. To create the construct coding this sensor, we used a plasmid coding the bioluminescent version of the sensor (described in (Sohn et al. 2018)), a kind gift from Tamas Balla (NICHD, NIH, Bethesda, USA). The D4H domain-coding sequence from this plasmid was subcloned into the pEYFP-C1 plasmid containing mVenus in place of EYFP, using Bglll and BamHI restriction enzymes. Cytosolic Cerulean was expressed from a pEYFP-N1 plasmid where EYFP had been replaced with Cerulean.

For fluorescent imaging, HEK293A cells (ATCC, USA) were maintained in Dulbecco's Modified Eagle Medium (DMEM - Lonza, Switzerland) complemented with 10\% fetal bovine serum (Biosera, France) and Penicillin/Streptomycin (100 U/ml and $100 \mu \mathrm{g} / \mathrm{ml}$, respectively - Lonza, Switzerland). Cells were seeded on poly-L-lysine pretreated $(0.001 \%, 1 \mathrm{~h}) 24$-well imaging plates (Eppendorf, Germany) at a density of $1 \mathrm{e} 05$ cells/well. On the next day, cells were co-transfected with plasmids coding cytoplasmic Cerulean and D4H-mVenus (0.25 $\mathrm{\mu g} /$ well each) using Lipofectamine 2000 (0.75 $\mu$ l/well, Invitrogen, USA).

Image acquisition started $24 \mathrm{~h}$ post-transfection, after the medium had been changed to 300 $\mu \mathrm{l} /$ well HEPES-buffered DMEM without phenol-red (Gibco, USA). Images were acquired automatically using the ImageXpress Micro Confocal High-Content Imaging System (Molecular Devices, USA), with a 40x Plan Fluor objective. CFP-2432C and fluorescein isothiocyanate (FITC) filter sets were used for Cerulean and D4H-mVenus images, respectively, both with an exposure time of $300 \mathrm{~ms}$. After acquiring control images (30 min), cells were treated with either 
DMSO (as control) or with the drugs indicated on Fig. 4E in a volume of $100 \mu \mathrm{l} /$ well (270 $\min$ ). Measurements were performed at $30^{\circ} \mathrm{C}$. Three independent measurements were made, with duplicate wells for each condition and 5 images/well taken for each time point.

All chemicals used for treatment were purchased from Sigma-Aldrich Merck (Germany). Amiodarone $\mathrm{HCl}$, chlorpromazine $\mathrm{HCl}$, loperamid $\mathrm{HCl}$ and rosuvastatin calcium were dissolved in DMSO, stored at $-20^{\circ} \mathrm{C}$ as $10 \mathrm{mM}$ stock solutions and diluted in cell medium promptly before cell treatment to a final concentration of $10 \mu \mathrm{M}$. M $\beta C D$ was stored as powder at $4^{\circ} \mathrm{C}$ and freshly dissolved in cell medium before treatment to a final concentration of $10 \mathrm{mM}$.

\section{Image analysis pipeline}

Images were segmented with Cellpose Python library (Stringer et al. 2021), which is a generalist, deep learning-based segmentation method. To select high-quality images the cytoplasm marker channel was used with Laplace filtering. We used high-quality images (filtered according to the upper threshold of Laplace value set to 3) as input of the Cellpose model, with parameter channel set to greyscale and cell diameter greater than 200 pixels.

After identifying cell boundaries, we applied binary erosion (scipy Python library (Virtanen et al. 2020)) with default structure and 10 iterations to determine cytoplasm boundary, or binary dilation with default structure and 5 iterations to determine PM outer boundary. The boundary of PM was determined by subtracting the cytoplasm boundary from the outer boundary. We calculated the $\log _{2}$ ratio of the mean PM and mean intracellular D4H fluorescence intensities for each cell in the $\mathrm{D} 4 \mathrm{H}$ channel to examine the changes of plasma membrane cholesterol distribution. For statistical analysis, we used log2(PM/IC) Time + Time : Drug + Exp linear model, where Time corresponds to elapsed time after drug treatment, Drug factor represents the used drug, using DMSO as reference level. Exp factor represents the $(n=3)$ individual experiments.

\section{Viral infection and cholesterol rescue experiments}

Amiodarone $\mathrm{HCl}$ (Sigma-Aldrich, Merck KGaA, Germany) was dissolved in DMSO (Sigma-Aldrich, Merck KGaA, Germany) and kept at $-20{ }^{\circ} \mathrm{C}$. Chlorpromazine (in house synthesized based on (Galons et al. 1985)) and loperamide $\mathrm{HCl}$ (Sigma-Aldrich, Merck KGaA, Germany) were freshly dissolved in water and filtered prior to the treatment. $10 \mathrm{mM}$ stock solutions were made from the drugs. Vero-E6 cells were seeded in a 96-well plate on the day before the experiments. On the next day the cells were treated with $100 \mu$ of $50 \mu \mathrm{M}$ remdesivir 
or loperamide or $12 \mu \mathrm{M}$ chlorpromazine or $6 \mu \mathrm{M}$ amiodarone solution overnight. 1 hour prior to the infection the cell culture media containing the different drugs was replaced with media containing $80 \mu \mathrm{M}$ cholesterol (Sigma-Aldrich, Merck KGaA, Germany). After the 1-hour-long cholesterol treatment the cells were infected with SARS-CoV-2 (GISAID accession ID: EPI_ISL_483637) at MOI:0.01 in a BSL-4 laboratory. Cells were incubated with the virus for 30 minutes then the media was replaced with fresh cell culture media. During the investigation (except cell seeding) DMEM (Lonza Group Ltd, Switzerland) supplemented with 1\% PenicillinStreptomycin (Lonza Group Ltd, Switzerland) and 2\% heat-inactivated fetal bovine serum (Gibco, Thermo Fisher Scientific Inc., MA, USA) was used. 48 hours post infection (hpi) the cells were inspected under microscope and RNA was extracted from the supernatant (Zybio EXM 3000 Nucleic Acid Isolation System, Nucleic Acid Extraction Kit B200-32). Viral copy number was determined using droplet- digital PCR technology (Bio-Rad Laboratories Inc., CA, USA). SARS-CoV-2 RdRp gene specific primers and probe were utilized (Forward: GTGARATGGTCATGTGTGGCGG, reverse: CARATGTTAAASACACTATTAGCATA and the probe was: FAM-CAGGTGGAACCTCATCAGGAGATGC-BBQ). For statistical analysis, measured viral copy numbers were log2 transformed, and we used a log2(CV) Drug * Cholesterol + Exp, where Drug factor represents the used drug (untreated as reference level), Cholesterol factor represents cholesterol replenishment treatment (no treatment as reference level). Exp factor corresponds to the $(n=4)$ individual experiments.

\section{Acknowledgements}

BS was supported by the Premium Postdoctoral Fellowship Program of the Hungarian Academy of Sciences (460044). DJT and PV were supported by the Hungarian Scientific Research Fund (OTKA K134357). On behalf of Project DRUGSENSPRED we thank for the usage of ELKH Cloud (https://science-cloud.hu/) that significantly helped us achieve the results published in this paper. The in vitro SARS-CoV-2 experiments were funded by the Hungarian Scientific Research Fund (OTKA KH129599), by the European Union and the European Social Fund (EFOP-3.6.1.-16-2016-00004), and by the Ministry for Innovation and Technology of Hungary (TUDFO/47138/2019-ITM). Schematic figures were created with BioRender.com.

We thank Aurélien Dugourd, Gergő Gulyás, Kinga Kovács and András D. Tóth for the helpful discussions regarding the manuscript, and Péter Mátyus for his help in organising the 
collaborations between computational and experimental groups. We thank Kata Szabolcsi for technical assistance in the cholesterol sensor experiments, Katalin Gombos, Zsófia Lanszki and Balázs Somogyi for their help in the in vitro infection experiments, and Tamás Kálai for compound synthesis.

\section{Code and data availability}

All analysis code to reproduce the results of this manuscript is available at https://github.com/comp-sys-pharm/SARS-CoV-2-cholesterol.

\section{Authors contributions}

SB and BS designed the research. SB, supervised by BS and $\mathrm{HL}$, performed the analysis and drafted the manuscript. AVU, supervised by JSR, performed CARNIVAL analysis. DJT, supervised by PV, performed cholesterol sensor experiments. HP, supervised by FJ, performed in vitro SARS-CoV-2 infection assay experiments. AK and MM contributed to infection assay experiments. BS contributed to manuscript writing and supervised the project. All authors read, commented, and approved the final manuscript.

\section{References}

Alvarez, Mariano J., Yao Shen, Federico M. Giorgi, Alexander Lachmann, B. Belinda Ding, B. Hilda Ye, and Andrea Califano. 2016. "Functional Characterization of Somatic Mutations in Cancer Using Network-Based Inference of Protein Activity." Nature Genetics 48 (8): 838-47.

Blanco-Melo, Daniel, Benjamin E. Nilsson-Payant, Wen-Chun Liu, Skyler Uhl, Daisy Hoagland, Rasmus Møller, Tristan X. Jordan, et al. 2020. "Imbalanced Host Response to SARS-CoV-2 Drives Development of COVID-19." Cell 181 (5): 1036-45.e9.

Bouhaddou, Mehdi, Danish Memon, Bjoern Meyer, Kris M. White, Veronica V. Rezelj, Miguel Correa Marrero, Benjamin J. Polacco, et al. 2020. "The Global Phosphorylation Landscape of SARS-CoV-2 Infection." Cell, June. https://doi.org/10.1016/j.cell.2020.06.034.

Chen, Bin, Li Ma, Hyojung Paik, Marina Sirota, Wei Wei, Mei-Sze Chua, Samuel So, and Atul J. Butte. 2017. "Reversal of Cancer Gene Expression Correlates with Drug Efficacy and Reveals Therapeutic Targets." Nature Communications 8 (July): 16022.

Chen, Fangyuan, Qingya Shi, Fen Pei, Andreas Vogt, Rebecca A. Porritt, Gustavo Garcia Jr, Angela C. Gomez, et al. 2021. "A Systems-Level Study Reveals Host-Targeted Repurposable Drugs against SARS-CoV-2 Infection." Molecular Systems Biology 17 (8): e10239.

Cuccarese, Michael F., Berton A. Earnshaw, Katie Heiser, Ben Fogelson, Chadwick T. Davis, Peter F. McLean, Hannah B. Gordon, et al. 2020. "Functional Immune Mapping with Deep-Learning Enabled Phenomics Applied to Immunomodulatory and COVID-19 Drug Discovery." https://doi.org/10.1101/2020.08.02.233064.

Daniloski, Zharko, Tristan X. Jordan, Hans-Hermann Wessels, Daisy A. Hoagland, Silva Kasela, Mateusz Legut, Silas Maniatis, et al. 2021. "Identification of Required Host Factors for SARS-CoV-2 Infection in Human Cells." Cell 184 (1): 92-105.e16. 
Dong, Ensheng, Hongru Du, and Lauren Gardner. 2020. "An Interactive Web-Based Dashboard to Track COVID-19 in Real Time." The Lancet Infectious Diseases 20 (5): 533-34.

Dugourd, A., and J. Saez-Rodriguez. 2019. "Footprint-Based Functional Analysis of Multi-Omic Data." Current Opinion in Systems Biology. https://www.sciencedirect.com/science/article/pii/S2452310019300149.

Ellinger, Bernhard, Denisa Bojkova, Andrea Zaliani, Jindrich Cinatl, Carsten Claussen, Sandra Westhaus, Oliver Keminer, et al. 2021. "A SARS-CoV-2 Cytopathicity Dataset Generated by High-Content Screening of a Large Drug Repurposing Collection." Scientific Data 8 (1): 70.

Galons, Herve, Marcel Miocque, Claude Combet-Farnoux, Younes Bensaid, Guy Decodts, and Georges Bram. 1985. "A Convenient Procedure for the Synthesis of Phenothiazine Drugs." Chemical \& Pharmaceutical Bulletin 33 (11): 5108-9.

Garcia-Alonso, Luz, Christian H. Holland, Mahmoud M. Ibrahim, Denes Turei, and Julio Saez-Rodriguez. 2019. "Benchmark and Integration of Resources for the Estimation of Human Transcription Factor Activities." Genome Research 29 (8): 1363-75.

Garcia-Alonso, Luz, Francesco lorio, Angela Matchan, Nuno Fonseca, Patricia Jaaks, Gareth Peat, Miguel Pignatelli, et al. 2018. "Transcription Factor Activities Enhance Markers of Drug Sensitivity in Cancer." Cancer Research 78 (3): 769-80.

Gordon, David E., Gwendolyn M. Jang, Mehdi Bouhaddou, Jiewei Xu, Kirsten Obernier, Kris M. White, Matthew J. O'Meara, et al. 2020. "A SARS-CoV-2 Protein Interaction Map Reveals Targets for Drug Repurposing." Nature, April. https://doi.org/10.1038/s41586-020-2286-9.

Heiser, Katie, Peter F. McLean, Chadwick T. Davis, Ben Fogelson, Hannah B. Gordon, Pamela Jacobson, Brett Hurst, et al. 2020. "Identification of Potential Treatments for COVID-19 through Artificial Intelligence-Enabled Phenomic Analysis of Human Cells Infected with SARS-CoV-2." bioRxiv. https://doi.org/10.1101/2020.04.21.054387.

Hoagland, Daisy A., Daniel J. B. Clarke, Rasmus Møller, Yuling Han, Liuliu Yang, Megan L. Wojciechowicz, Alexander Lachmann, et al. 2020. "Modulating the Transcriptional Landscape of SARS-CoV-2 as an Effective Method for Developing Antiviral Compounds." bioRxiv. https://doi.org/10.1101/2020.07.12.199687.

Holland, Christian H., Bence Szalai, and Julio Saez-Rodriguez. 2019. "Transfer of Regulatory Knowledge from Human to Mouse for Functional Genomics Analysis." Biochimica et Biophysica Acta, Gene Regulatory Mechanisms, September, 194431.

Horton, Jay D., Joseph L. Goldstein, and Michael S. Brown. 2002. "SREBPs: Activators of the Complete Program of Cholesterol and Fatty Acid Synthesis in the Liver." The Journal of Clinical Investigation 109 (9): 1125-31.

Jeon, Sangeun, Meehyun Ko, Jihye Lee, Inhee Choi, Soo Young Byun, Soonju Park, David Shum, and Seungtaek Kim. n.d. "Identification of Antiviral Drug Candidates against SARS-CoV-2 from FDA-Approved Drugs." https://doi.org/10.1101/2020.03.20.999730.

Josset, Laurence, Vineet D. Menachery, Lisa E. Gralinski, Sudhakar Agnihothram, Pavel Sova, Victoria S. Carter, Boyd L. Yount, Rachel L. Graham, Ralph S. Baric, and Michael G. Katze. 2013. "Cell Host Response to Infection with Novel Human Coronavirus EMC Predicts Potential Antivirals and Important Differences with SARS Coronavirus." mBio 4 (3): e00165-13.

Kindrachuk, Jason, Britini Ork, Brit J. Hart, Steven Mazur, Michael R. Holbrook, Matthew B. Frieman, Dawn Traynor, et al. 2015. "Antiviral Potential of ERK/MAPK and PI3K/AKT/mTOR Signaling Modulation for Middle East Respiratory Syndrome Coronavirus Infection as Identified by Temporal Kinome Analysis." Antimicrobial Agents and Chemotherapy 59 (2): 1088-99.

Konrat, Robert, Henrietta Papp, Valéria Szijártó, Tanja Gesell, Gábor Nagy, Mónika Madai, Safia Zeghbib, et al. 2020. "The Anti-Histamine Azelastine, Identified by Computational Drug Repurposing, Inhibits SARS-CoV-2 Infection in Reconstituted Human Nasal Tissue In Vitro." bioRxiv. https://doi.org/10.1101/2020.09.15.296228.

Kunkel, Steven D., Manish Suneja, Scott M. Ebert, Kale S. Bongers, Daniel K. Fox, Sharon E. Malmberg, Fariborz Alipour, Richard K. Shields, and Christopher M. Adams. 2011. "mRNA Expression Signatures of Human Skeletal Muscle Atrophy Identify a Natural Compound That Increases Muscle Mass." Cell Metabolism 13 (6): 627-38.

Laise, Pasquale, Gideon Bosker, Xiaoyun Sun, Yao Shen, Eugene F. Douglass, Charles Karan, Ronald B. 
Realubit, Sergey Pampou, Andrea Califano, and Mariano J. Alvarez. 2020. “The Host Cell ViroCheckpoint: Identification and Pharmacologic Targeting of Novel Mechanistic Determinants of Coronavirus-Mediated Hijacked Cell States." bioRxiv. https://doi.org/10.1101/2020.05.12.091256.

Lei, Xiaobo, Xiaojing Dong, Ruiyi Ma, Wenjing Wang, Xia Xiao, Zhongqin Tian, Conghui Wang, et al. 2020. "Activation and Evasion of Type I Interferon Responses by SARS-CoV-2." Nature Communications 11 (1): 3810.

Li, Chengjun, Armand Bankhead 3rd, Amie J. Eisfeld, Yasuko Hatta, Sophia Jeng, Jean H. Chang, Lauri D. Aicher, et al. 2011. "Host Regulatory Network Response to Infection with Highly Pathogenic H5N1 Avian Influenza Virus." Journal of Virology 85 (21): 10955-67.

Liu, Anika, Panuwat Trairatphisan, Enio Gjerga, Athanasios Didangelos, Jonathan Barratt, and Julio Saez-Rodriguez. 2019. "From Expression Footprints to Causal Pathways: Contextualizing Large Signaling Networks with CARNIVAL." NPJ Systems Biology and Applications 5 (November): 40.

Love, Michael I., Wolfgang Huber, and Simon Anders. 2014. "Moderated Estimation of Fold Change and Dispersion for RNA-Seq Data with DESeq2." Genome Biology 15 (12): 550.

Maekawa, Masashi. 2017. "Domain 4 (D4) of Perfringolysin O to Visualize Cholesterol in Cellular Membranes-The Update.” Sensors 17 (3). https://doi.org/10.3390/s17030504.

Maekawa, Masashi, and Gregory D. Fairn. 2015. "Complementary Probes Reveal That Phosphatidylserine Is Required for the Proper Transbilayer Distribution of Cholesterol." Journal of Cell Science 128 (7): 1422-33.

Malcomson, Beth, Hollie Wilson, Eleonora Veglia, Gayathri Thillaiyampalam, Ruth Barsden, Shauna Donegan, Amal El Banna, et al. 2016. "Connectivity Mapping (ssCMap) to Predict A20-Inducing Drugs and Their Antiinflammatory Action in Cystic Fibrosis." Proceedings of the National Academy of Sciences of the United States of America 113 (26): E3725-34.

McCallum, Matthew, Jessica Bassi, Anna De Marco, Alex Chen, Alexandra C. Walls, Julia Di lulio, M. Alejandra Tortorici, et al. 2021. "SARS-CoV-2 Immune Evasion by the B.1.427/B.1.429 Variant of Concern.” Science 373 (6555): 648-54.

Napolitano, Francesco, Gennaro Gambardella, Diego Carrella, Xin Gao, and Diego di Bernardo. 2020. "Computational Drug Repositioning and Elucidation of Mechanism of Action of Compounds against SARS-CoV-2." arXiv [q-bio.GN]. arXiv. http://arxiv.org/abs/2004.07697.

Olbei, Marton, Isabelle Hautefort, Dezso Modos, Agatha Treveil, Martina Poletti, Lejla Gul, Claire D. Shannon-Lowe, and Tamas Korcsmaros. 2021. "SARS-CoV-2 Causes a Different Cytokine Response Compared to Other Cytokine Storm-Causing Respiratory Viruses in Severely III Patients." Frontiers in Immunology 12 (March): 629193.

Pedregosa, Fabian, Gaël Varoquaux, Alexandre Gramfort, Vincent Michel, Bertrand Thirion, Olivier Grisel, Mathieu Blondel, et al. 2011. "Scikit-Learn: Machine Learning in Python." Journal of Machine Learning Research: JMLR 12 (Oct): 2825-30.

Pushpakom, Sudeep, Francesco lorio, Patrick A. Eyers, K. Jane Escott, Shirley Hopper, Andrew Wells, Andrew Doig, et al. 2019. "Drug Repurposing: Progress, Challenges and Recommendations." Nature Reviews. Drug Discovery 18 (1): 41-58.

Ragab, Dina, Haitham Salah Eldin, Mohamed Taeimah, Rasha Khattab, and Ramy Salem. 2020. "The COVID-19 Cytokine Storm; What We Know So Far." Frontiers in Immunology 11 (June): 1446.

Ramana, C. V., N. Grammatikakis, M. Chernov, H. Nguyen, K. C. Goh, B. R. Williams, and G. R. Stark. 2000. "Regulation of C-Myc Expression by IFN-Gamma through Stat1-Dependent and -Independent Pathways." The EMBO Journal 19 (2): 263-72.

Rehwinkel, Jan, and Michaela U. Gack. 2020. "RIG-I-like Receptors: Their Regulation and Roles in RNA Sensing." Nature Reviews. Immunology 20 (9): 537-51.

Ritchie, Matthew E., Belinda Phipson, Di Wu, Yifang Hu, Charity W. Law, Wei Shi, and Gordon K. Smyth. 2015. "Limma Powers Differential Expression Analyses for RNA-Sequencing and Microarray Studies." Nucleic Acids Research 43 (7): e47.

Riva, Laura, Shuofeng Yuan, Xin Yin, Laura Martin-Sancho, Naoko Matsunaga, Sebastian Burgstaller, Lars Pache, et al. 2020. "A Large-Scale Drug Repositioning Survey for SARS-CoV-2 Antivirals." bioRxiv. https://doi.org/10.1101/2020.04.16.044016.

Sanders, David W., Chanelle C. Jumper, Paul J. Ackerman, Dan Bracha, Anita Donlic, Hahn Kim, Devin Kenney, et al. 2021. "SARS-CoV-2 Requires Cholesterol for Viral Entry and Pathological Syncytia 
Formation." eLife 10 (April). https://doi.org/10.7554/eLife.65962.

Schubert, Michael, Bertram Klinger, Martina Klünemann, Anja Sieber, Florian Uhlitz, Sascha Sauer, Mathew J. Garnett, Nils Blüthgen, and Julio Saez-Rodriguez. 2018. "Perturbation-Response Genes Reveal Signaling Footprints in Cancer Gene Expression." Nature Communications 9 (1): 20.

Selinger, Christian, Jennifer Tisoncik-Go, Vineet D. Menachery, Sudhakar Agnihothram, G. Lynn Law, Jean Chang, Sara M. Kelly, Pavel Sova, Ralph S. Baric, and Michael G. Katze. 2014. "Cytokine Systems Approach Demonstrates Differences in Innate and pro-Inflammatory Host Responses between Genetically Distinct MERS-CoV Isolates." BMC Genomics 15 (December): 1161.

Seth, Rashu B., Lijun Sun, and Zhijian J. Chen. 2006. "Antiviral Innate Immunity Pathways." Cell Research 16 (2): 141-47.

$\mathrm{Si}$, Longlong, Haiqing Bai, Melissa Rodas, Wuji Cao, Crystal Yuri Oh, Amanda Jiang, Rasmus Moller, et al. 2020. "Human Organ Chip-Enabled Pipeline to Rapidly Repurpose Therapeutics during Viral Pandemics." https://doi.org/10.1101/2020.04.13.039917.

Sims, Amy C., Susan C. Tilton, Vineet D. Menachery, Lisa E. Gralinski, Alexandra Schäfer, Melissa M. Matzke, Bobbie-Jo M. Webb-Robertson, et al. 2013. "Release of Severe Acute Respiratory Syndrome Coronavirus Nuclear Import Block Enhances Host Transcription in Human Lung Cells." Journal of Virology 87 (7): 3885-3902.

Sirota, Marina, Joel T. Dudley, Jeewon Kim, Annie P. Chiang, Alex A. Morgan, Alejandro Sweet-Cordero, Julien Sage, and Atul J. Butte. 2011. "Discovery and Preclinical Validation of Drug Indications Using Compendia of Public Gene Expression Data." Science Translational Medicine 3 (96): 96ra77.

Sohn, Mira, Marek Korzeniowski, James P. Zewe, Rachel C. Wills, Gerald R. V. Hammond, Jana Humpolickova, Lukas Vrzal, et al. 2018. "PI(4,5)P2 Controls Plasma Membrane PI4P and PS Levels via ORP5/8 Recruitment to ER-PM Contact Sites." The Journal of Cell Biology 217 (5): 1797-1813.

Stathias, Vasileios, Anna M. Jermakowicz, Marie E. Maloof, Michele Forlin, Winston Walters, Robert K. Suter, Michael A. Durante, et al. 2018. "Drug and Disease Signature Integration Identifies Synergistic Combinations in Glioblastoma." Nature Communications 9 (1): 5315.

Stringer, Carsen, Tim Wang, Michalis Michaelos, and Marius Pachitariu. 2021. "Cellpose: A Generalist Algorithm for Cellular Segmentation." Nature Methods 18 (1): 100-106.

Subramanian, Aravind, Rajiv Narayan, Steven M. Corsello, David D. Peck, Ted E. Natoli, Xiaodong Lu, Joshua Gould, et al. 2017. "A Next Generation Connectivity Map: L1000 Platform and the First 1,000,000 Profiles." Cell 171 (6): 1437-52.e17.

Szalai, Bence, and Julio Saez-Rodriguez. 2020. "Why Do Pathway Methods Work Better than They Should?" FEBS Letters 594 (24): 4189-4200.

Szalai, Bence, Vigneshwari Subramanian, Christian H. Holland, Róbert Alföldi, László G. Puskás, and Julio Saez-Rodriguez. 2019. "Signatures of Cell Death and Proliferation in Perturbation Transcriptomics Data-from Confounding Factor to Effective Prediction." Nucleic Acids Research 47 (19): 10010-26.

Touret, Franck, Magali Gilles, Karine Barral, Antoine Nougairède, Jacques van Helden, Etienne Decroly, Xavier de Lamballerie, and Bruno Coutard. 2020. "In Vitro Screening of a FDA Approved Chemical Library Reveals Potential Inhibitors of SARS-CoV-2 Replication." Scientific Reports 10 (1): 13093.

Tummino, Tia A., Veronica V. Rezelj, Benoit Fischer, Audrey Fischer, Matthew J. O’Meara, Blandine Monel, Thomas Vallet, et al. 2021. "Drug-Induced Phospholipidosis Confounds Drug Repurposing for SARS-CoV-2." Science 373 (6554): 541-47.

Türei, Dénes, Alberto Valdeolivas, Lejla Gul, Nicolàs Palacio-Escat, Michal Klein, Olga Ivanova, Márton Ölbei, et al. 2021. "Integrated Intra- and Intercellular Signaling Knowledge for Multicellular Omics Analysis." Molecular Systems Biology 17 (3): e9923.

Virtanen, Pauli, Ralf Gommers, Travis E. Oliphant, Matt Haberland, Tyler Reddy, David Cournapeau, Evgeni Burovski, et al. 2020. "SciPy 1.0: Fundamental Algorithms for Scientific Computing in Python." Nature Methods 17 (3): 261-72.

Wang, Manli, Ruiyuan Cao, Leike Zhang, Xinglou Yang, Jia Liu, Mingyue Xu, Zhengli Shi, Zhihong Hu, Wu Zhong, and Gengfu Xiao. 2020. "Remdesivir and Chloroquine Effectively Inhibit the Recently Emerged Novel Coronavirus (2019-nCoV) in Vitro." Cell Research 30 (3): 269-71.

Wang, Shaobo, Wanyu Li, Hui Hui, Shashi Kant Tiwari, Qiong Zhang, Ben A. Croker, Stephen Rawlings, Davey Smith, Aaron F. Carlin, and Tariq M. Rana. 2020. "Cholesterol 25-Hydroxylase Inhibits 
SARS-CoV-2 and Other Coronaviruses by Depleting Membrane Cholesterol." The EMBO Journal 39 (21): e106057.

Weston, Stuart, Christopher M. Coleman, Rob Haupt, James Logue, Krystal Matthews, and Matthew B.

Frieman. 2020. "Broad Anti-Coronaviral Activity of FDA Approved Drugs against SARS-CoV-2 in Vitro and SARS-CoV in Vivo." https://doi.org/10.1101/2020.03.25.008482.

Wyler, Emanuel, Kirstin Mösbauer, Vedran Franke, Asija Diag, Lina Theresa Gottula, Roberto Arsiè,

Filippos Klironomos, et al. 2021. "Transcriptomic Profiling of SARS-CoV-2 Infected Human Cell Lines Identifies HSP90 as Target for COVID-19 Therapy." iScience 24 (3): 102151.

York, Autumn G., Kevin J. Williams, Joseph P. Argus, Quan D. Zhou, Gurpreet Brar, Laurent Vergnes,

Elizabeth E. Gray, et al. 2015. "Limiting Cholesterol Biosynthetic Flux Spontaneously Engages Type I IFN Signaling." Cell 163 (7): 1716-29.

Zhou, Yadi, Yuan Hou, Jiayu Shen, Yin Huang, William Martin, and Feixiong Cheng. 2020.

"Network-Based Drug Repurposing for Novel Coronavirus 2019-nCoV/SARS-CoV-2." Cell Discovery 6 (March): 14.

\section{Supplementary Materials}

\section{Loperamide}

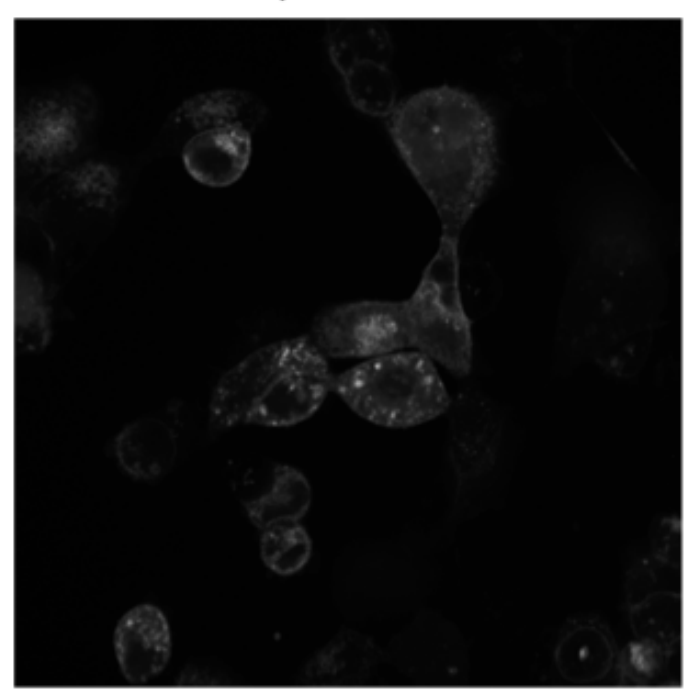

Rosuvastatin

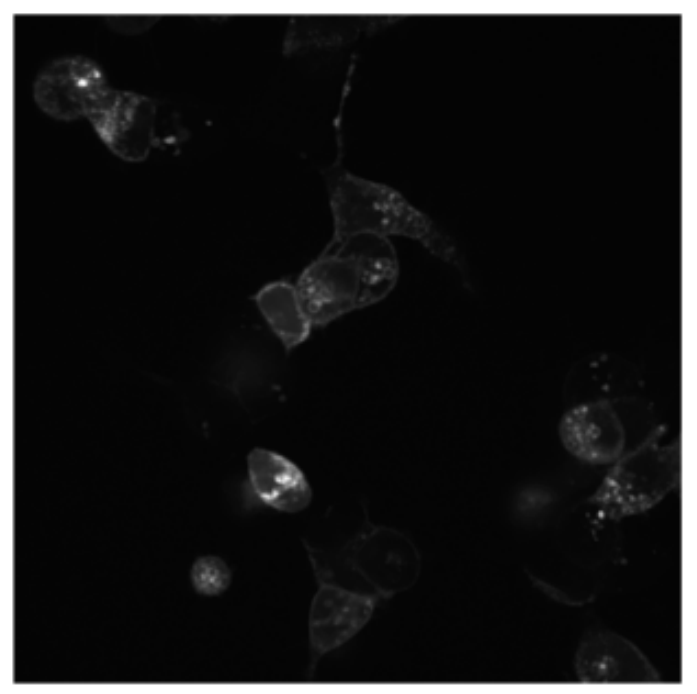

Supplementary Figure 1. Representative confocal microscopy images of D4H-mVenus transfected HEK293A cells treated with loperamide and rosuvastatin. 

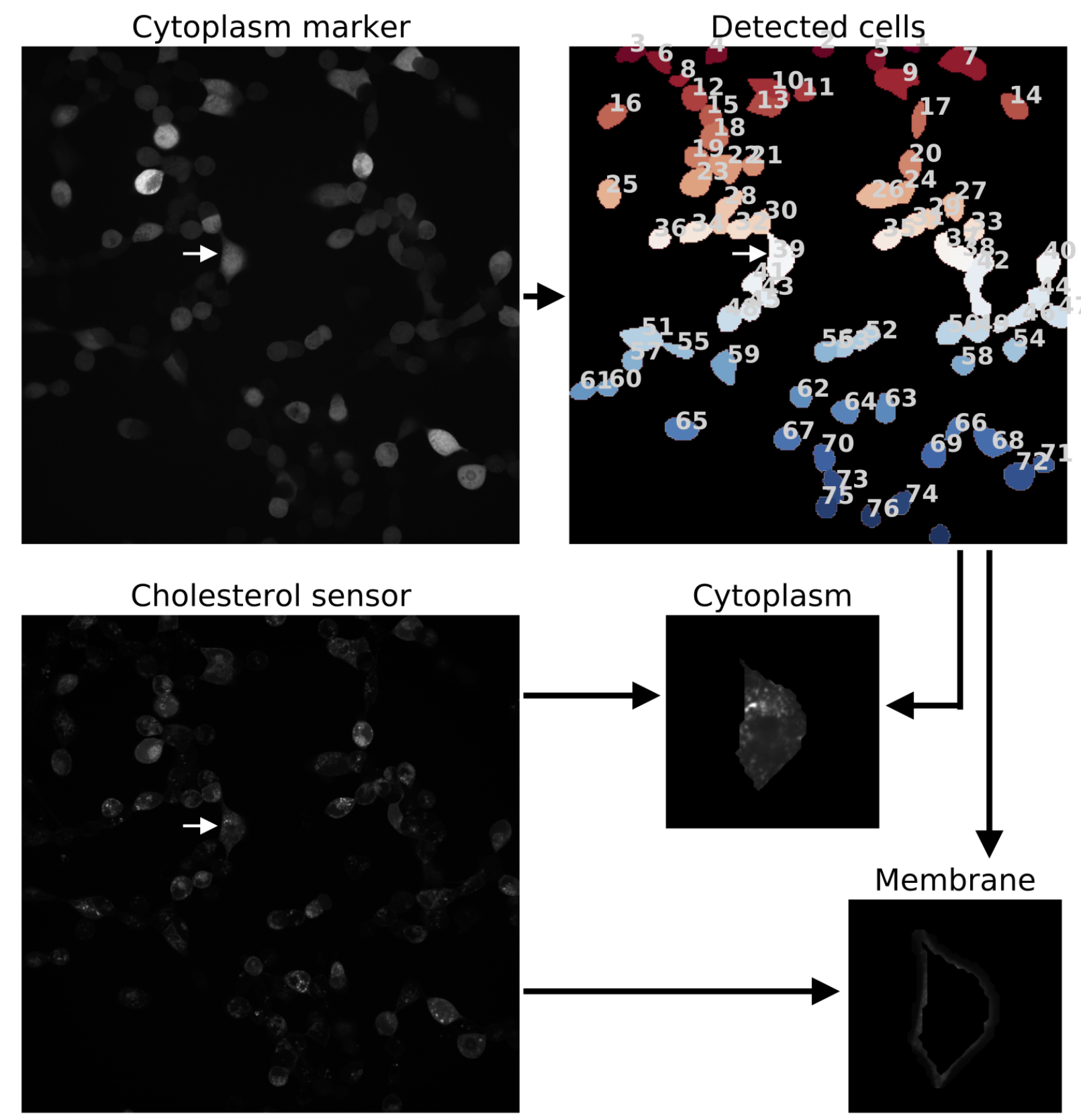

Supplementary Figure 2. Determination of cytoplasm and membrane boundaries. Cells are detected on the cytoplasm marker channel, then boundaries of cytoplasm and membrane are determined for each cell. The $D 4 H$ channel is used for the calculation of the PM/IC ratio. 
bioRxiv preprint doi: https://doi.org/10.1101/2021.09.10.459786: this version posted September 10. 2021. The copvriaht holder for this preprint (which was not certified by peer review) is the author/funder, who has granted bioRxiv a license to display the preprint in perpetuity. It is made available under aCC-BY 4.0 International license.

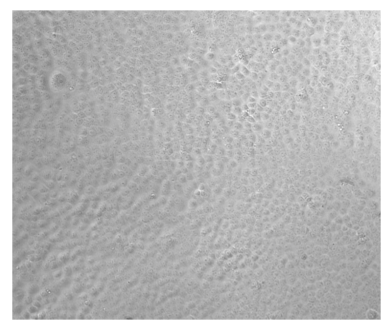

Control

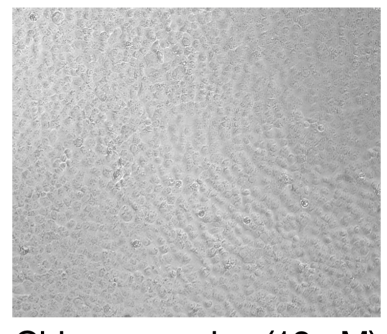

Chlorpromazine $(12 \mu \mathrm{M})$

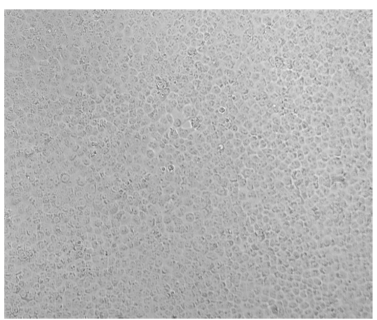

Amiodarone $(6 \mu \mathrm{M})$

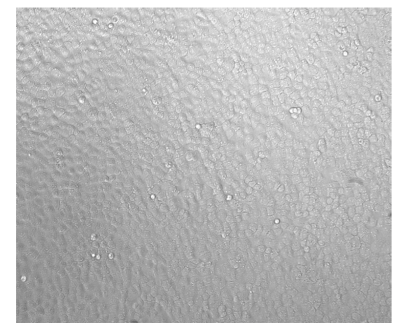

Loperamide $(50 \mu \mathrm{M})$

Supplementary Figure 3. Absence of marked toxic effects of used drugs in the tested concentrations. 